\title{
Sirt3 Deficiency Shortens Life Span and Impairs Cardiac Mitochondrial Function Rescued by Opa1 Gene Transfer
}

\author{
Ariela Benigni, ${ }^{1,{ }^{*}}$ Paola Cassis, ${ }^{1, *}$ Sara Conti, ${ }^{1, *}$ Luca Perico,, Daniela Corna, ${ }^{1}$ Domenico Cerullo, \\ Lorena Zentilin, ${ }^{2}$ Carlamaria Zoja, ${ }^{1}$ Annalisa Perna, ${ }^{1}$ Vincenzo Lionetti, ${ }^{3,4}$ Mauro Giacca, ${ }^{2,5}$ \\ Piera Trionfini,, Susanna Tomasoni, and Giuseppe Remuzzi ${ }^{1,6}$
}

\begin{abstract}
Aims: Sirtuins, a family of $\mathrm{NAD}^{+}$-dependent deacetylases, are recognized as nondispensable regulators of aging processes. Sirtuin 3 (SIRT3) is the main mitochondrial deacetylase that maintains mitochondrial bioenergetics, an essential prerequisite for healthy aging. In this study, using Sirt3 knockout $\left(\operatorname{Sirt} 3^{-/}\right)$mice, we sought to establish whether Sirt3 deficiency affected life span, an endpoint that has never been tested formally in mammals, and uncover the mechanisms involved in organ damage associated with aging.

Results: Sirt ${ }^{-/-}$mice experienced a shorter life span than wild-type mice and severe cardiac damage, characterized by hypertrophy and fibrosis, as they aged. No alterations were found in organs other than the heart. Sirt3 deficiency altered cardiac mitochondrial bioenergetics and caused hyperacetylation of optic atrophy 1 (OPA1), a SIRT3 target. These changes were associated with aberrant alignment of trans-mitochondrial cristae in cardiomyocytes, and cardiac dysfunction. Gene transfer of deacetylated Opal restored cristae alignment in Sirt $3^{-/-}$mice, ameliorated cardiac reserve capacity, and protected the heart against hypertrophy and fibrosis. The translational relevance of these findings is in the data showing that SIRT3 silencing in human-induced pluripotent stem cell-derived cardiomyocytes led to mitochondrial dysfunction and altered contractile phenotype, both rescued by Opal gene transfer.

Innovation: Our findings indicate that future approaches to heart failure could include SIRT3 as a plausible therapeutic target.

Conclusion: SIRT3 has a major role in regulating mammalian life span. Sirt3 deficiency leads to cardiac abnormalities, due to defective trans-mitochondrial cristae alignment and impaired mitochondrial bioenergetics. Correcting cardiac OPA1 hyperacetylation through gene transfer diminished heart failure in Sirt ${ }^{-/-}$mice during aging. Antioxid. Redox Signal. 31, 1255-1271.
\end{abstract}

Keywords: SIRT3, mammalian life span, heart failure, trans-mitochondrial cristae alignment, mitochondrial bioenergetics, gene transfer

\footnotetext{
${ }^{1}$ Istituto di Ricerche Farmacologiche Mario Negri IRCCS, Centro Anna Maria Astori, Science and Technology Park Kilometro Rosso, Bergamo, Italy.

${ }^{2}$ Molecular Medicine Laboratory, International Centre for Genetic Engineering and Biotechnology (ICGEB), Trieste, Italy.

${ }^{3}$ Institute of Life Sciences, Scuola Superiore Sant'Anna, Pisa, Italy.

${ }^{4}$ UOS Anesthesia and Intensive Care, Fondazione Toscana "G. Monasterio", Pisa, Italy.

${ }^{5}$ Department of Medical, Surgical and Health Sciences, University of Trieste, Trieste, Italy.

${ }^{6}$ L. Sacco' Department of Biomedical and Clinical Sciences, University of Milan, Milan, Italy.

*These three authors contributed equally to this work.
} 


\section{Innovation}

Sirtuin 3 (Sirt3) deficiency is associated with several diseases of aging, but whether the sequelae of Sirt3 deficiency translated into an effect on life span is unknown. We show that Sirt $^{-1-}$ mice have shortened life span with cardiac abnormalities that include altered mitochondrial bioenergetics and hyperacetylation of optic atrophy 1 (OPA1), associated with aberrant alignment of transmitochondrial cristae in cardiomyocytes, and cardiac dysfunction. Given our finding that the correction of cardiac OPA1 hyperacetylation through gene transfer restored trans-mitochondrial cristae alignment and limited heart failure in aged $\mathrm{Sirt}^{-/}$mice, we envision future approaches to heart failure, including SIRT3 as a plausible therapeutic target.

\section{Introduction}

IRTUINs (SIRTs) are an evolutionary conserved family of $\checkmark \mathrm{NAD}^{+}$-dependent deacetylases that regulate multiple cellular processes, including stress resistance, apoptosis, inflammation, and mitochondrial energy homeostasis, and are recognized as critical determinants in counteracting the aging process and age-related diseases $(19,27)$.

In mammals, three members of the SIRT family - sirtuin 3 (SIRT3), SIRT4, and SIRT5-are localized in the mitochondrial matrix and modulate mitochondrial metabolism, but it is SIRT3 that functions uniquely as a major regulator of the whole organelle acetylome $(33,42)$. In fact, in mice the deletion of Sirt3, but not of Sirt4 or Sirt5, resulted in striking global hyperacetylation of mitochondrial proteins (25), indicating that SIRT3 is central to mitochondrial deacetylase activity, which is an essential prerequisite for healthy aging (52). Indeed, almost $60 \%$ of mitochondrial proteins can be deacetylated quickly to meet cellular metabolic demands and contribute substantially to maintaining bioenergetic adaptation during aging (16).

In this context, it has been demonstrated that SIRT3 finetunes the process of energy production, due to its primary role in fatty acid oxidation through the deacetylation of longchain acyl-CoA dehydrogenase (17), as well as the activation of mitochondrial enzymatic chain proteins, including complexes I (2), II (14), and V (39), which are involved in oxidative phosphorylation through which ATP is generated.

In addition to energy supply and nutrient oxidation, SIRT3 affects other major aspects of mitochondrial biology, including reactive oxygen species detoxification and mitochondrial dynamics $(6,31,35)$. Sirt3 attenuation or ablation is known to be associated with the accelerated development of several diseases of aging, including cancer and cardiovascular, renal, hepatic, and neurodegenerative disorders (5, $18,20,29,32,34,43)$. Features that are characteristic of aging are found in the myocardium of Sirt3 heart-specific knockout mice, such as mitochondrial protein dysfunction, enhanced oxidative stress, and impaired energy metabolism (24). Whether sequelae of SIRT3 deficiency translated into an effect on life span was not addressed.

In this study, we wanted (i) to investigate the impact that the lack of Sirt3 had on life span in mice, which, surprisingly, had not been studied in mammals yet; (ii) to establish which organ is primarily affected by Sirt3 deficiency during aging, and identify the molecular mechanisms underlying organ dysfunction in aged mice; (iii) to mitigate target organ dysfunction therapeutically; and (iv) to demonstrate the translational relevance of these findings by studying SIRT3-silenced human-induced pluripotent stem cell (iPSC)-derived cardiomyocytes in vitro.

\section{Results}

\section{Sirt3 deficiency shortens life span and promotes cardiac dysfunction}

We set out to examine the impact of Sirt3 gene disruption in male mice compared with wild type (WT) control littermates. All Sirt3 knockout animals died within 34 months, at which time $28 \%$ of their control littermates were still alive (Fig. 1). Log-rank test analysis showed significant differences in the survival curves between Sirt $^{---}$and WT mice. Compared with WT littermates, the median life span of Sirt $^{-/-}$mice was $19 \%$ shorter (median $=50$ th percentile: from 32 to 26 months) ( $p$-value of $<0.0001$, Fig. 1). Compared with WT littermates, the maximum life span of Sirt $3^{-/}$ mice (i.e., the life span of the oldest $10 \%$ of a cohort to die) was $10 \%$ shorter (90th percentile: from 35.5 to 32 months), a $p$-value of 0.011 .

Of all the organs analyzed in aged mice in the present study, the heart was the main organ affected by Sirt3 deficiency. Aged Sirt $3^{-/}$mice developed severe cardiac damage, characterized by hypertrophy and fibrosis, which is in line with previous studies $(15,45,46)$. An increased heart to body weight ratio (Fig. 2A) and the accumulation of collagen deposition in the interstitial space (Fig. 2B, C) were observed in aged Sirt $^{-/-}$but not in WT mice.

A similar number of immune cells were found in the cardiac tissue of Sirt $3^{-/-}$mice and WT mice (neutrophils: $5.5 \pm$ 0.4 vs. $4.4 \pm 0.6$; macrophages: $0.5 \pm 0.2$ vs. $0.8 \pm 0.1$; $\mathrm{CD}^{+}$ T cells: $0.9 \pm 0.1$ vs. $0.6 \pm 0.2$; and $\mathrm{CD}^{+} \mathrm{T}$ cells: $0.8 \pm 0.2$ vs. $0.6 \pm 0.2$, expressed as number of cells/field in $n=6$ mice/ group), which ruled out the possibility that an immune or inflammatory reaction caused the cardiac changes in Sirt $3^{-/-}$ mice. Cardiac abnormalities in these animals were not associated with changes in lipid plasma levels or renal function impairment in terms of proteinuria (Supplementary Table S1).

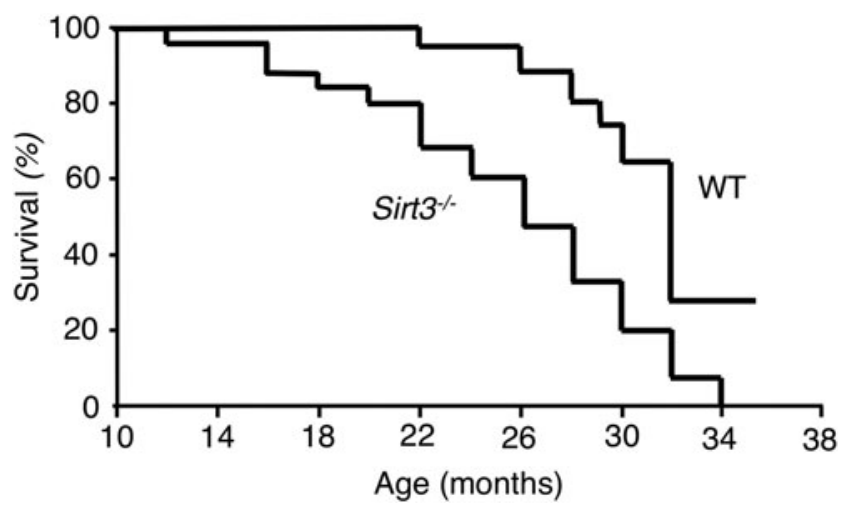

FIG. 1. Sirt3 $3^{-/-}$mice have reduced life span. KaplanMeier survival curves for male WT (right censored) and Sirt $3^{-/-}$mice. The $p$-value was derived from log-rank calculations, $n=25$ mice/group. $p$-value of $<0.0001$ versus WT. SIRT3, sirtuin 3; WT, wild type. 
A

FIG. 2. Sirt $^{-/-}$mice exhibit cardiac abnormalities. (A) Quantification of HW and $\mathrm{BW}$ ratio $(\mathrm{HW} / \mathrm{BW})$ in aged $\mathrm{WT}$ and Sirt $3^{-/-}$mice (20-35.5 months of age, $n=8 /$ group). (B) Collagen deposition in the hearts of aged mice (20-35.5 months of age, $n=8$ /group). (C) Representative images of collagen deposition assessed by Sirius Red staining in cardiac tissue harvested from aged WT and Sirt $3^{-1}$ mice. Scale bars: $20 \mu \mathrm{m}$. Values are mean \pm SEM. The $p$-values were derived from unpaired Student's $t$-test. $* * p<0.01$; $* p<0.05$. BW, body weight; HW, heart weight; SEM, standard error of the mean.
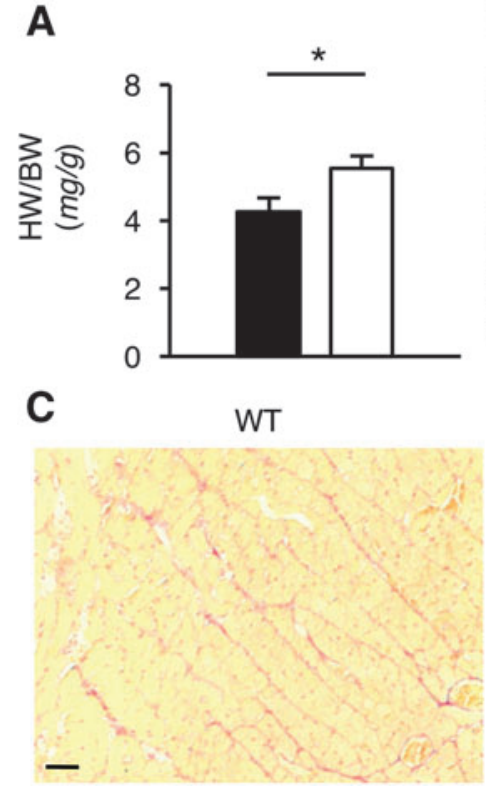
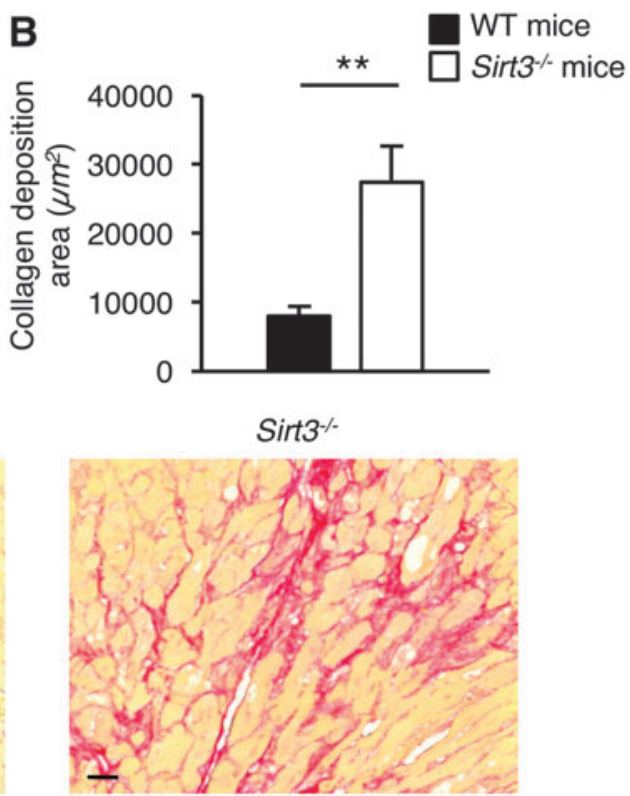

As for the other organs, mild renal interstitial fibrosis developed in Sirt $3^{-/}$mice comparable with WT mice, as shown by stainings of the kidney tissue for collagen I and III fibers (Sirius Red, Supplementary Fig. S1) or for alpha-smooth muscle actin ( $\alpha$-SMA, Supplementary Fig. S1). However, these abnormalities did not translate into a pathological renal phenotype (Supplementary Table S1). As for the liver, we found modest abnormalities that did not translate into fullblown liver dysfunction, as demonstrated by normal levels of plasma alanine aminotransferase (ALT) and aspartate aminotransferase (AST) levels (Supplementary Table S1). No histological alterations were found in the brain, nor was an increased incidence of tumors observed in Sirt $3^{-/-}$mice compared with WT mice.

\section{Sirt3 deficiency compromises mitochondrial connectivity and communication in the heart}

The absolute mass of cardiac mitochondria was significantly reduced in Sirt $^{-/-}$compared with WT mice, which was demonstrated in the analysis of citrate synthase activity (Fig. 3A). In addition to mass reduction, the ATP synthesis rate was also reduced in Sirt $3^{-/-}$hearts compared with those of WT mice, reflecting low energy production in the former group (Fig. 3B).

Next, we wanted to understand why the energetic state of Sirt $3^{-1-}$ mice was compromised. We thus turned our attention to mitochondrial connectivity and communication, a crucial factor in cellular homeostasis (21). We investigated the structural integrity of the mitochondrial network in Sirt3 $3^{-/-}$ and WT mice using transmission electron microscopy at a nanometer resolution range.

We found that Sirt3 deficiency affected trans-mitochondrial cristae coordination to a significant extent. Specifically, we observed an aberrant alignment of trans-mitochondrial cristae in most mitochondria in the hearts of mice lacking Sirt3, compared with age-matched WT mice (Fig. 3C). Mitochondrial cristae architecture, although not completely disrupted, was predominantly abnormal in Sirt $3^{-/-}$mice compared with the regular cristae distribution in WT mice (Fig. 3C). Specifically, mitochondrial cristae were rarely organized as parallel stacks, as is usually observed in WT animals (Fig. 3C).

\section{Effect of Opa1 gene transfer on mitochondrial structure and function}

There is evidence that SIRT3 regulates mitochondrial function and dynamics through the deacetylation and activation of optic atrophy 1 (OPA1), a pro-fusion protein of the inner mitochondrial membrane $(31,41)$. In this study, we wondered whether the lack of trans-mitochondrial cristae coordination in our model was caused by Sirt3 deficiency and the consequent hyperacetylation of OPA1. Total OPA1 was evaluated in isolated mitochondria of cardiac tissue by Western blot analysis. We found that total OPA1 was significantly reduced in Sirt $3^{-/}$compared with WT mice (Fig. 3D), and it was hyperacetylated (Fig. 3D).

We then investigated whether the impaired transmitochondrial cristae alignment found in Sirt $3^{-1-}$ mice could be corrected by inducing the expression of the deacetylated and active mutant (mut) form of OPA1. To this end, we generated an adeno-associated virus serotype 9 (AAV9)mutOpal-vector encoding for a deacetylation-mimetic version of murine OPA1, in which two conserved lysines, $\mathrm{K}^{944}$ and $\mathrm{K}^{949}$, were replaced with two $\operatorname{arginins}(\mathrm{R})$ to mimic the function of SIRT3. AAV9 was chosen for its uniquely exuberant cardiac tropism (55). An AAV9-LacZ vector served as a control.

Cardiomyocytes were efficiently transduced after the injection of $1.3 \times 10^{12}$ viral genome/animal of AAV9-LacZ (Supplementary Fig. S2). Western blot analysis of mitochondrial extracts revealed that gene transfer of mutOpal increased total OPA1 protein levels, as expected, and effectively reduced the quantity of acetylated OPA 1 in $S i r t 3^{-/-}$ hearts (Fig. 4A). Two weeks after injection, the expression of the SIRT3-insensitive version of OPA1 did not have an impact on cardiac mitochondrial mass (citrate synthase activity, $\mu \mathrm{mole} / \mathrm{mL} / \mathrm{min}$ : $0.014 \pm 0.003$ in Sirt $^{-/-}$mice injected with 
A
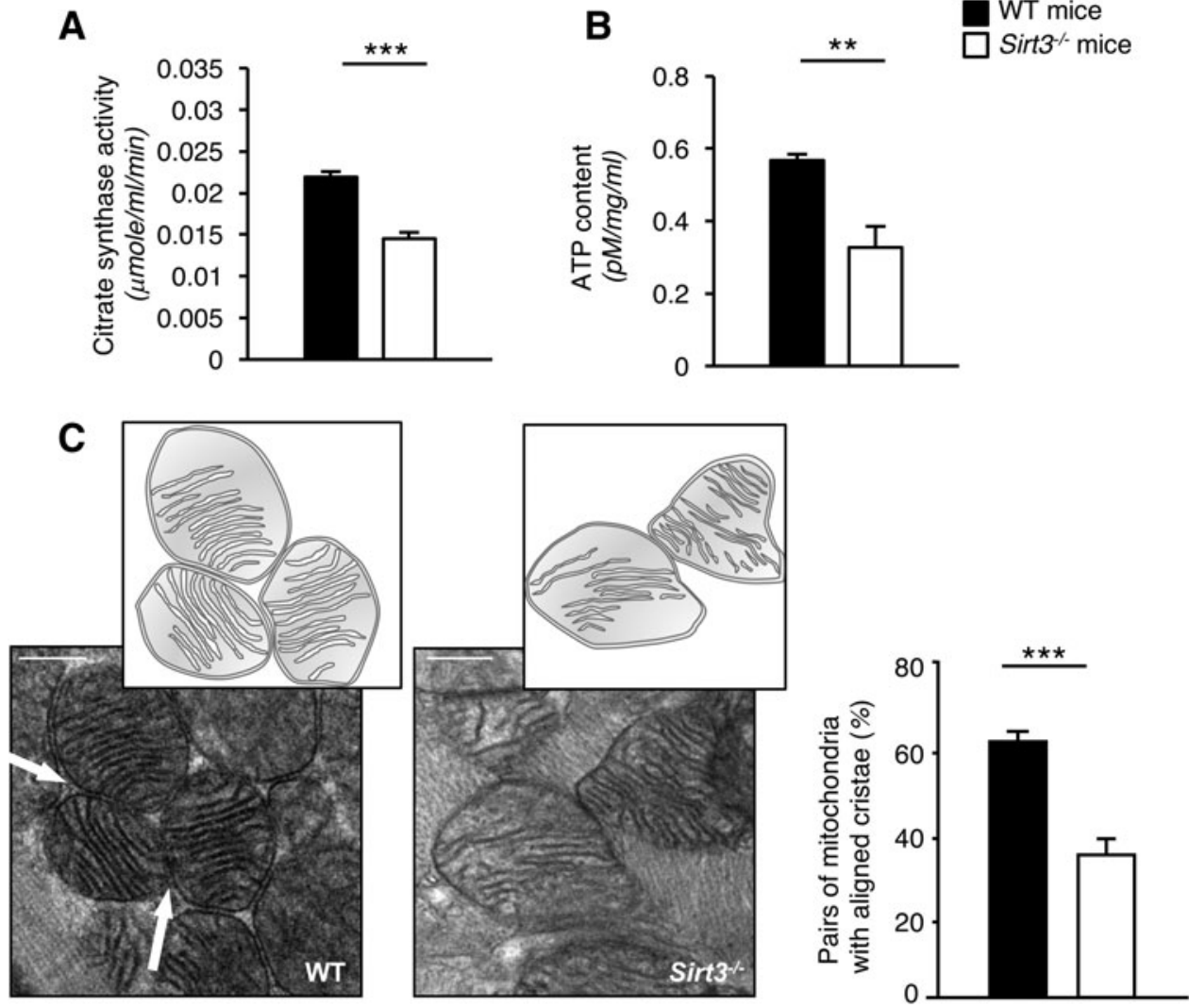

D
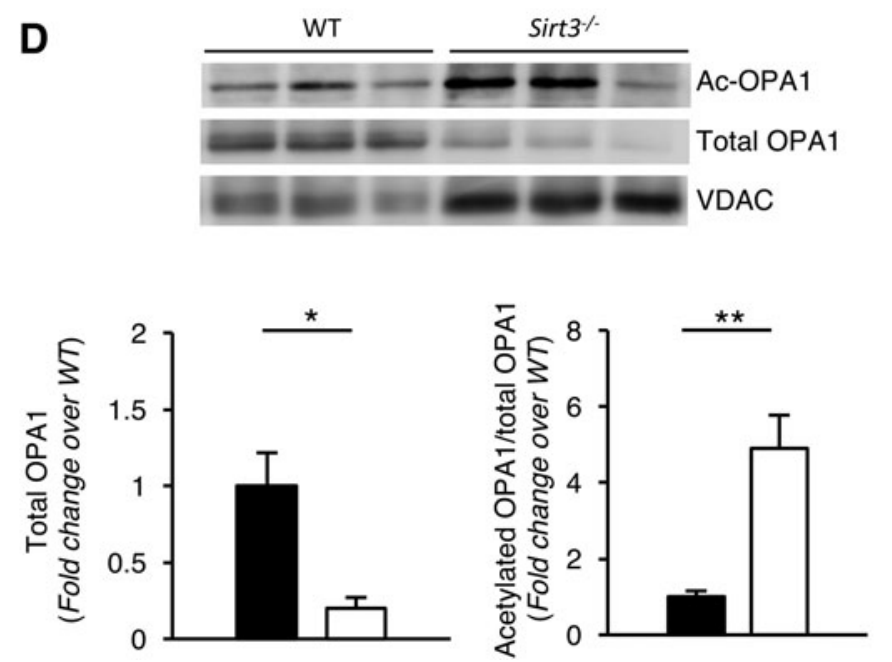

FIG. 3. Alterations in mitochondrial bioenergetics, trans-mitochondrial cristae alignment, and OPA1 acetylation in cardiac tissue of Sirt3 $^{-/-}$mice. (A) Levels of citrate synthase activity, and (B) ATP content in total cardiac extracts of WT and Sirt $3^{-/}$mice ( $n=4 /$ group). (C) Representative transmission electron micrographs and percentage of mitochondria with aligned transmitochondrial cristae in WT and Sirt $^{-\prime}$ mice $(n=3 /$ group). Scale bar: $1 \mu \mathrm{m}$. Insets: schematic drawing of trans-mitochondrial coordination of cristae. White arrows illustrate the representative events of trans-mitochondrial cristae alignment between adjacent mitochondria. (D) Representative gels and densitometric analysis of Western blot of acetylated (ac-OPA1) and total OPA1 in mitochondrial cardiac extracts from WT and Sirt $3^{-/}$mice ( $n=4 /$ group). VDAC was used as sampleloading controls in isolated mitochondria. WT and Sirt $3^{-1-}$ mice were analyzed at 15 months of age. Values are mean \pm SEM. For (A, B, D), the $p$-values were derived from unpaired Student's $t$-test. For (C), the $p$-value was derived from GENMOD procedure in $\mathrm{SAS}^{\circledR}$. $* * * p<0.001 ; \quad * * p<0.01$; $* p<0.05$. OPA1, optic atrophy 1; VDAC, voltagedependent anion channel.
AAV9-LacZ vs. $0.013 \pm 0.002$ in Sirt $^{-/-}$mice injected with AAV9-mutOpal, $n=4$ mice/group), but improved cardiac ATP content in Sirt3-deficient mice (Fig. 4B). Strikingly, mutOpal gene delivery restored the proper alignment of the cristae of adjacent mitochondria (Fig. 4C, D). Transfection with mutOpal also effectively reduced cardiac hypertrophy (Fig. 4E).

To confirm that the protective effect of gene transfer on cardiac abnormalities was fully dependent on OPA1's deacetylated status rather than on its expression levels, we performed gene transfer experiments of WT Opal (wtOpal) in Sirt $3^{-/}$mice. Total OPA1 protein levels increased after gene delivery (Fig. 4F). Under Sirt3-deficient conditions, wtOpal could not be deacetylated as revealed by comparable levels of hyperacetylated OPA1 in mice injected with AAV9-wtOpal and AAV9-LacZ (Fig. 4F). Likewise, the transduction of wtOpal did not improve cardiac mitochondrial mass (citrate synthase activity, $\mu \mathrm{mole} / \mathrm{mL} / \mathrm{min}: 0.016 \pm 0.001$ in Sirt $^{-/-}$ mice injected with AAV9-LacZ vs. $0.015 \pm 0.001$ in Sirt $3^{-/-}$ mice injected with AAV9-wtOpal, $n=4$ mice/group), or ATP content (Fig. 4G). Unlike mutOpal, wtOpal did not promote trans-mitochondrial cristae alignment (Fig. 4C, D), nor did it limit cardiac hypertrophy (Fig. 4E).

\section{Effect of Opa1 gene transfer on cardiac dysfunction in the long term}

Next, we investigated whether the effects that the gene transfer of the mutOpal had on mitochondrial structure and 
function, observed earlier, translated into end-organ cardiac protection.

To determine the optimal timing for therapeutic intervention, we first evaluated $\alpha$-SMA expression and collagen I deposition as markers of fibrosis in the cardiac tissue of Sirt $3^{-/-}$mice (Supplementary Fig. S3A). $\alpha$-SMA expression was already higher in Sirt $3^{-/-}$mice compared with WT mice at 15 months (\% of positive cells/field: $6.78 \pm 0.29 v s$. $3.60 \pm 0.96, p$-value of $<0.05, n=3 \mathrm{mice} /$ group) and rose further at 20 months $(15.27 \pm 3.53 v s .3 .62 \pm 0.19$, $p$-value of $<0.05, n=3 \mathrm{mice} / g r o u p)$ (Supplementary Fig. S3A). Collagen I deposition was similar in Sirt $3^{-/-}$and WT mice at 15 months (score: $0.20 \pm 0.03$ vs. $0.17 \pm 0.02, n=3$ mice/group), but rose significantly higher at 20 months in Sirt $^{-\gamma-}$ mice than in WT mice $(0.56 \pm 0.05$ vs. $0.23 \pm 0.03, p$-value of $<0.01, n=3$ mice/group) (Supplementary Fig. S3B). Based on $\alpha$-SMA and collagen I expression in cardiac tissue, Sirt $3^{-/-}$ mice at 18-20 months of age were treated with AAV9-mutOpal or AAV9-LacZ and sacrificed after 3 months. The gene transfer approach proved capable of preserving in the long term an effective state of OPA1 deacetylation in Sirt $3^{-/-}$ mice (Fig. 5A).

Since respiratory chain complexes are key structures for mitochondrial bioenergetics and reside on the cristae membrane, we evaluated the activity of the gatekeeper of the respiratory chain, Complex I, the formation and stabilization of which are dependent on OPA1. The activity of immunocaptured Complex I from cardiac tissue lysates was analyzed based on oxidation of NADH to $\mathrm{NAD}^{+}$, and found to be remarkably lower in Sirt $3^{-/-}$mice receiving AAV9LacZ vector compared with WT mice (Fig. 5B). MutOpal transduction restored the Complex I activity to levels that were indistinguishable from those of WT mice (Fig. 5B). As a consequence, $\mathrm{NAD}^{+}$levels, which were reduced in AAV9$\mathrm{LacZ} \mathrm{Sirt}^{-/-}$mice, were normalized after mutOpal overexpression (Fig. 5C).

The reduced respiratory capacity of AAV9-LacZ Sirt3 ${ }^{-/-}$ mice yielded lower ATP production, which was replenished to control levels by mutOpal (Fig. 5D). The improvement in mitochondrial bioenergetics by mutOpal was accompanied by an increase in mitochondrial mass, evaluated as citrate synthase activity (Fig. 5E).

Long-term transduction with mutOpal led to a reduction in hypertrophy and the heart-to-body weight ratio was normalized (Fig. 6A). The protective effect of mutOpal on cardiac hypertrophy was accompanied by reduced fibrosis, assessed through both $\alpha$-SMA (Fig. 6B) and collagen I (Fig. 6C) immunostainings.

We then measured global left ventricular function using echocardiography at rest and during a low-dose dobutamine (LDD) stress test to evaluate myocardial viability and contractile performance in response to an acute increase in workload (48). LDD stress echocardiography was tolerated well in all animals and we did not encounter any complications. The dobutamine was effective at a low dose, as demonstrated by a statistically significant increase in left ventricle fractional shortening (LVFS), under stress, compared with baseline in WT mice (Fig. 6D). In Sirt $3^{-/}$mice receiving AAV9-LacZ vector, the LDD stress test did not increase the inotropic cardiac reserve, which instead increased significantly in Sirt $3^{-/-}$mice transduced with mutOpal (Fig. 6D). There was no difference in chronotropic response to LDD between groups (AAV9-LacZ: pre $515.0 \pm 34.1$ beats per minute (bpm) vs. post $474.3 \pm 34.7 \mathrm{bpm}$; AAV9-mutOpal: pre $449.8 \pm 8.8 \mathrm{bpm} v s$. post $459.0 \pm 5.9 \mathrm{bpm}$; WT: pre $474.4 \pm$ $40.3 \mathrm{bpm} v s$. post $536.6 \pm 60.9 \mathrm{bpm})$. This is not surprising, since others have found that LDD did not induce changes in heart rate (8). All the above findings suggest a link between the replacement of trans-mitochondrial cristae alignment and recovery of cardiac structure and function.

That the effect of gene transfer actually depended on OPA1 deacetylation was supported by findings that hyperacetylation of total mitochondrial proteins, including the SIRT3 target superoxide dismutase 2 (SOD2), in Sirt $3^{-/-}$ mice (Supplementary Fig. S4A) was not affected by mutOpal gene delivery (Supplementary Fig. S4B).

\section{SIRT3 silencing alters mitochondrial morphology and contractile behavior in human iPSC-derived cardiomyocytes}

Having established that there is a strong correlation between SIRT3 and cardiac function in mice, we sought to investigate the effect of SIRT3 deficiency on spontaneously beating human iPSC-derived cardiomyocytes. To this end, human iPSC-derived cardiomyocytes were silenced for the SIRT3 gene through a specific small interfering RNA (siRNA). SIRT3 silencing resulted in $85 \%$ inhibition of SIRT3 mRNA expression, compared with cells transfected with small interfering RNA null (siNULL) (Fig. 7A).

In this setting, we investigated the effect of SIRT3 deletion on mitochondrial structural integrity by using an image preprocessing tool to quantitatively capture morphological changes in the mitochondrial network (50). Using MitoTracker staining, we found that almost the $60 \%$ of siNULL iPSC-derived cardiomyocytes exhibited a subset of highly packed and round mitochondria near the perinuclear region, while highly packed mitochondria were found in only $20 \%$ of SIRT3-deficient iPSC-derived cardiomyocytes (Fig. 7B).

In addition, iPSC-derived cardiomyocytes exhibited a highly interconnected mitochondrial network, which extends through the whole cytoplasmic compartment of the cells following siNULL transfection (Fig. 7C). The deletion of SIRT3 markedly altered the overall structural architecture of the mitochondrial lattice in iPSC-derived cardiomyocytes, to the extent that the mitochondrial networks and area were significantly reduced compared with their SIRT3-competent counterpart (Fig. 7C). In-depth analysis revealed that the reduced mitochondrial network, which followed SIRT3 silencing, was the consequence of the specific loss of both mitochondrial branches (siNULL: $551 \pm 29 v s$. small interfering RNA for sirtuin 3 [siSIRT3]: $371 \pm 20$ number/cell, $p$-value of $<0.01$ ) and junctions (siNULL: $186 \pm 9$ vs. siSIRT3: $129 \pm 8$ number/cell, $p$-value of $<0.01)$, as described by Valente et al. (50).

An extensive body of evidence has shown that both the shape and positioning of mitochondria tightly controls $\mathrm{Ca}^{2+}$ handling of cardiomyocytes (47). To investigate the mechanisms underlying the observed altered beating contraction, we analyzed the $\mathrm{Ca}^{2+}$-handling properties of iPSC-derived cardiomyocytes by using the $\mathrm{Ca}^{2+}$ fluorescent dye FLUO-4. The live-imaging recording showed that after siNULL transfection, high-intensity $\mathrm{Ca}^{2+}$ sparks emerged synchronously in both the nuclear and cytoplasmic regions of the 
iPSC-derived cardiomyocytes (Supplementary Movie S1). In contrast, SIRT3 silencing translated into irregular $\mathrm{Ca}^{2+}$ release that was not synchronized among iPSC-derived cardiomyocytes of a monolayer, without apparent changes in either intensity or duration of $\mathrm{Ca}^{2+}$ sparks (Supplementary Movie S2).

We subsequently examined how SIRT3 silencing affected cardiomyocyte contraction. Through Cell Observer analysis, phase-contrast monitoring of living human iPSC-derived cardiomyocytes treated with siNULL revealed a regular, autonomous beating rate with a frequency that remained almost constant during the temporal course of the measurements (Supplementary Movie S3). In the face of reduced SIRT3 expression, iPSC-derived cardiomyocytes exhibited uncontrolled spontaneous beating that led to the generation of ectopic foci of contraction (Supplementary Movie S4).

To evaluate whether the dysfunctioned phenotype of cardiomyocytes could be rescued by restoring functional OPA1, we transduced mutOpal in SIRT3-silenced iPSC-derived cardiomyocytes. First, we found that SIRT3-silenced iPSCderived cardiomyocytes exhibited downregulation of OPA1 and increased protein acetylation compared with siNULLtransfected cells (Fig. 7D, E). By contrast, mutOpal gene transfer effectively increased total OPA 1 protein expression and reduced its acetylation levels (Fig. 7D, E). To investigate whether the restoration of functional OPA1 translated into an amelioration of cardiomyocyte function, we assessed human iPSC-derived cardiomyocyte contraction. Cardiomyocytes transfected with siNULL and LacZ exhibited a regular, autonomous beating rate (Supplementary Movie S5), while SIRT3-silenced cardiomyocytes transfected with LacZ showed uncontrolled spontaneous beating (Supplementary Movie S6). Remarkably, transfection of mutOpal restored the spontaneous synchronous beating of SIRT3-silenced cardiomyocytes (Supplementary Movie S7).

To provide further functional data on mitochondrial activity in cardiomyocytes transfected with mutOpal, we evaluated the expression of ATP synthase subunit e (ATP5I) that is required for ATP synthase dimerization and proper functioning (38). As shown in Figure 7D and F, we found that SIRT3 silencing in iPSC-derived cardiomyocytes significantly reduced ATP5I protein expression compared with siNULL-transfected cells. Levels of ATP5I protein were even higher than in siNULL-transfected cells by mutOpal gene transfer (Fig. 7D, F).

\section{Discussion}

In this study, we report for the first time that SIRT3 has a major role in regulating life span in mice. The limited survival time of Sirt $3^{-/}$animals is associated with severe heart structure damage and dysfunction. Heart damage parallels defective trans-mitochondrial cristae alignment and impaired bioenergetics, which are linked to suboptimal OPA1 deacetylation. The salient feature of the present study is the utilization of a targeted gene transfer involving mutOpal that reverses heart failure. Silencing SIRT3 in human iPSCderived cardiomyocytes disrupts mitochondrial integrity and alters cell contractility, rescued by mutOpal gene delivery.

The first novel finding of this study is that Sirt3 deficiency shortens life span in mice. To the best of our knowledge, there are no other studies that document the effect of a specific mitochondrial SIRT on survival in mammals. A study that has been recently published (53) is worth mentioning here. In Drosophila melanogaster, life span is shortened by knockdown of Sirt4, encoding the sole predicted mitochondrial SIRT in this model organism, while flies overexpressing Sirt4 ubiquitously or in the fat body are long-lived. It is noteworthy that several phenotypes of Sirt4 knockout fruit fly are reminiscent of those found here in Sirt $3^{-/}$mice. As the authors of the above article have indicated, it is possible that Sirt4 in the fruit fly could be a counterpart of the mammalian SIRT3.

In this study, we have demonstrated that Sirt3-deficient animals primarily experience signs of severe cardiomyopathy as they age. While this finding may not be entirely new (15, 45 ), it is worth mentioning here that $\operatorname{Sirt}^{-/-}$mice were followed up for a long period of time, long enough to demonstrate differences in heart pathologies, including hypertrophy and fibrosis. In the long run, these changes were associated with signs of heart failure.

As the main mitochondrial SIRT, SIRT3 deacetylates many proteins in the organelles and promotes diverse aspects of health span, but the role of SIRT3 goes beyond deacetylating specific mitochondrial substrates. It also regulates mitochondrial function at many other levels (26). Given that cardiac tissue has the highest number of mitochondria and the

FIG. 4. Effect of mutOpal and wtOpal gene transfer on OPA1 acetylation, mitochondrial bioenergetics, transmitochondrial cristae alignment, and cardiac hypertrophy in Sirt3 ${ }^{-1-}$ mice. (A) Representative gels and densitometric analysis of Western blot of acetylated (ac-OPA1) and total OPA1 in mitochondrial cardiac extracts of Sirt $3^{-/-}$mice injected with AAV9-LacZ (LacZ) or AAV9-mutOpal (mutOpal) ( $n=4 /$ group). VDAC was used as sample-loading controls in isolated mitochondria. (B) Levels of ATP content in total cardiac extracts of Sirt3 ${ }^{-1-}$ mice injected with LacZ or mutOpal ( $n=4$ /group). (C) Representative transmission electron micrographs and (D) percentage of mitochondria with aligned transmitochondrial cristae in Sirt $3^{-/}$mice injected with LacZ, mutOpal, or wtOpal ( $n=3 /$ group). Scale bar: $1 \mu \mathrm{m}$. Insets: schematic drawing of trans-mitochondrial coordination of cristae. White arrows indicate adjacent mitochondria with aligned trans-mitochondrial cristae. (E) Quantification of cardiac hypertrophy in WT and Sirt3 ${ }^{-1}$ mice injected with LacZ, mutOpal, or wtOpal ( $n=4$ /group). (F) Representative gels and densitometric analysis of Western blot of acetylated (ac-OPA1) and total OPA1 in mitochondrial cardiac extracts of Sirt $3^{-1-}$ mice injected with LacZ ( $n=4 /$ group) or AAV9-wtOpal (wtOpal) ( $n=3$ /group). VDAC was used as sample-loading controls in isolated mitochondria. (G) Levels of ATP content in total cardiac extracts of Sirt $3^{-/-}$mice injected with LacZ or wtOpal ( $n=4 /$ group). Gene transfer experiments were performed in Sirt $3^{-/-}$mice at 15 months of age, and the analyses were performed 2 weeks after AAV9 injection. Values are mean \pm SEM. For $(\mathbf{A}, \mathbf{B}$ and $\mathbf{F}, \mathbf{G})$, the $p$-values were derived from unpaired Student's $t$-test. For (D), the $p$-value was derived from GENMOD procedure in SAS. For $(\mathbf{E})$, the $p$-values were derived from ANOVA with Tukey post hoc test. $* * * p<0.001 ; * * p<0.01 ; * p<0.05$. AAV9, adeno-associated virus serotype 9; ANOVA, analysis of variance. 
A
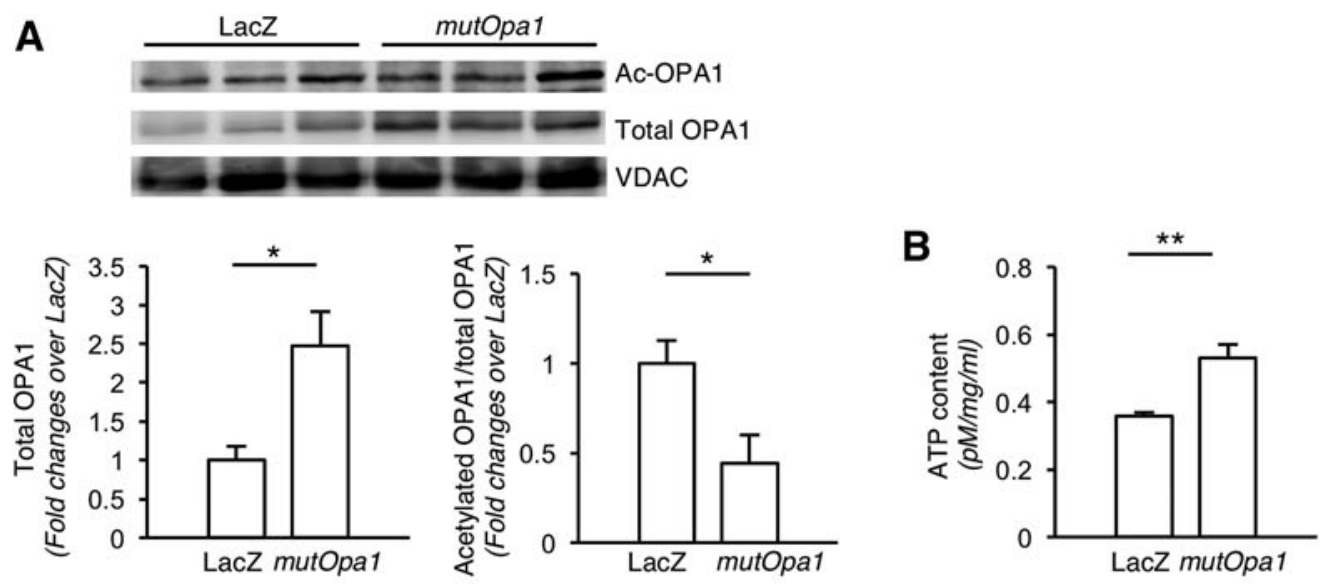

C
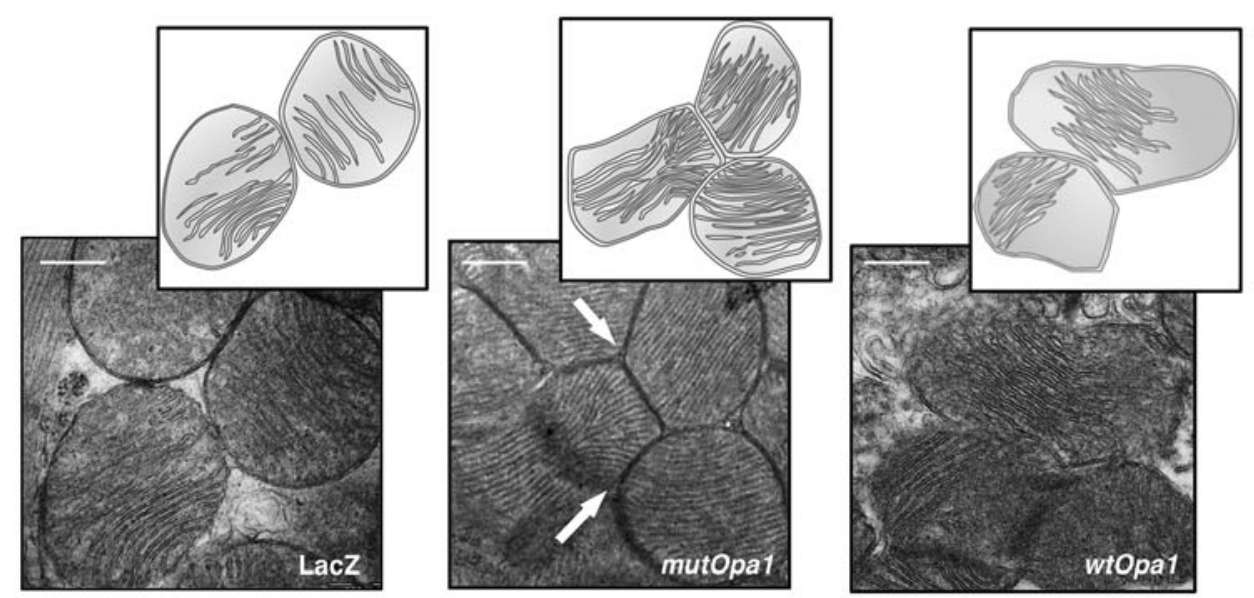

D

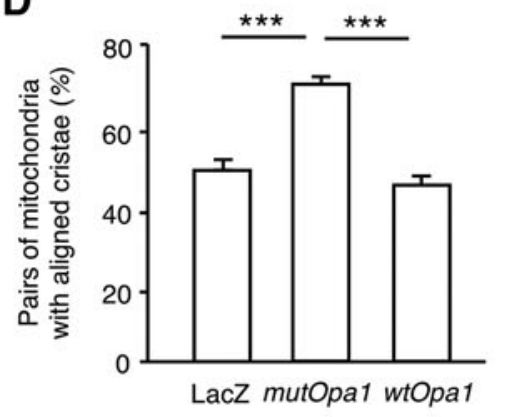

E



F


G

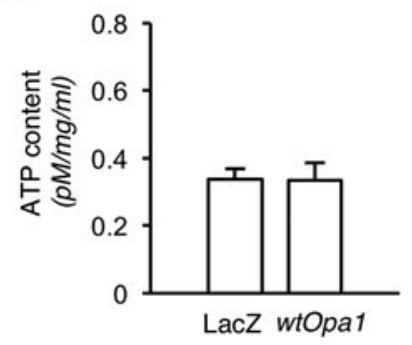



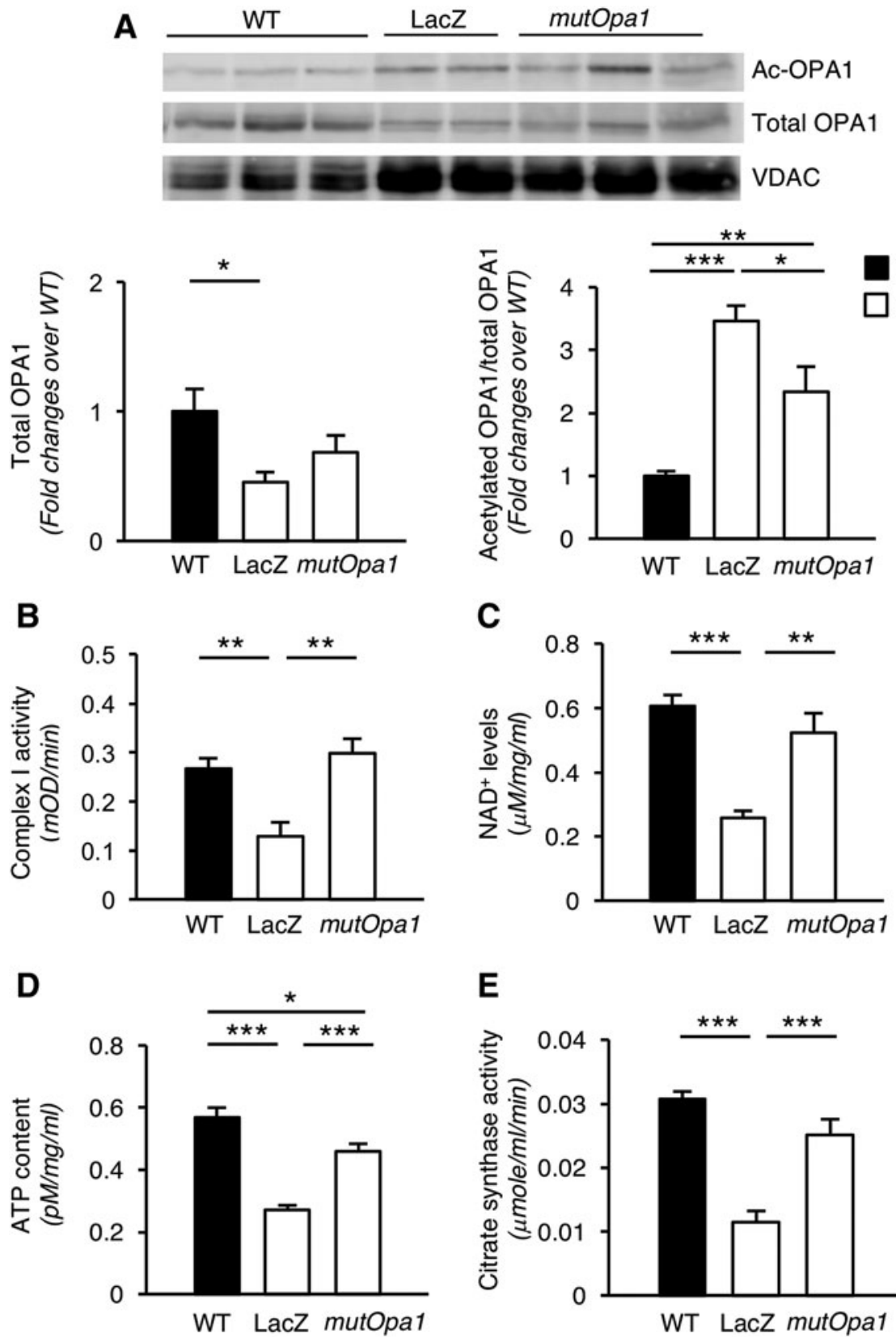

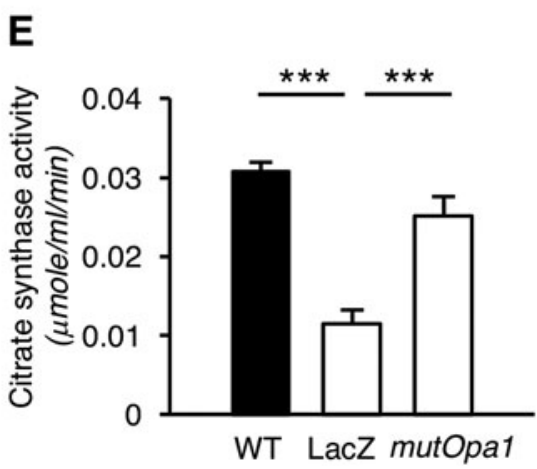

FIG. 5. Long-term effect of mutOpal gene transfer on mitochondrial function in Sirt3 ${ }^{-/-}$mice. (A) Representative gels and densitometric analysis of Western blot of acetylated (ac-OPA1) and total OPA1 in mitochondrial cardiac extracts of WT mice, and Sirt $3^{-/-}$mice injected with LacZ or mutOpal ( $n=6$ /group). VDAC was used as sample-loading controls in isolated mitochondria. (B-E) Evaluation of Complex I enzymatic activity (B) expressed as milli optical density per min (mOD/min), NAD ${ }^{+}$levels (C), ATP content (D), and citrate synthase activity (E) in cardiac extracts of WT mice, and Sirt $3^{--}$mice injected with LacZ or mutOpal ( $n=6$ /group). Gene transfer experiments were performed in Sirt3 $3^{-1-}$ mice at 18-20 months of age, and the analyses were performed 3 months after AAV9 injection. WT age-matched mice were studied as controls. Values are mean \pm SEM. The $p$-values were derived from ANOVA with Tukey post hoc test, $* * * p<0.001$; $* * p<0.01$; and $* p<0.05$. highest levels of oxygen consumption of all organs, Sirt3 deletion primarily affects the heart. Indeed, Sirt $3^{-/-}$mice exhibited abnormal cardiac mitochondrial clustering and reduced mass associated with a lower than normal ATP synthesis rate, which would suggest an altered bioenergetic state.

Actually, bioenergetics in mitochondria depends on the reciprocal connectivity, which is guaranteed by specific junctions acting as unique electrochemical systems $(3,7,36)$. While we confirmed the elegant findings of Picard et al., which found that adjacent mitochondria finely coordinate the curvature of their cristae with each other (37), we also found that in Sirt $3^{-/-}$mice, this parallel alignment with the cristae of neighboring mitochondria was lost. This could explain the compromised mitochondrial energetics in Sirt3-deficient animals in the present study. To date, no other instance of aberrant trans-mitochondrial cristae alignment in a disease model has been reported. It is tempting to speculate that shortened life span and heart abnormalities could indeed be direct consequences of abnormal intermitochondrial interaction.

Since mitochondrial dysfunction in the hypertrophic heart is dependent on the state of deacetylation of OPA1 (41), we chose to investigate whether the lack of coordination of mitochondrial cristae was due to suboptimal OPA1 deacetylation of Sirt $3^{-/}$animals. Gene transfer experiments with constitutively deacetylated mutOpal fully reconstituted mitochondrial cristae coordination with the appropriate curvature within mitochondria in $\operatorname{Sirt}^{-/-}$mice. This unique property of OPA1 has never been reported before, to the best of our knowledge.

We also found that the restoration of mitochondrial connectivity translated functionally into recovery of ATP content, which can be taken as an indication of restored mitochondrial bioenergetics. The possible role that mutOpal 

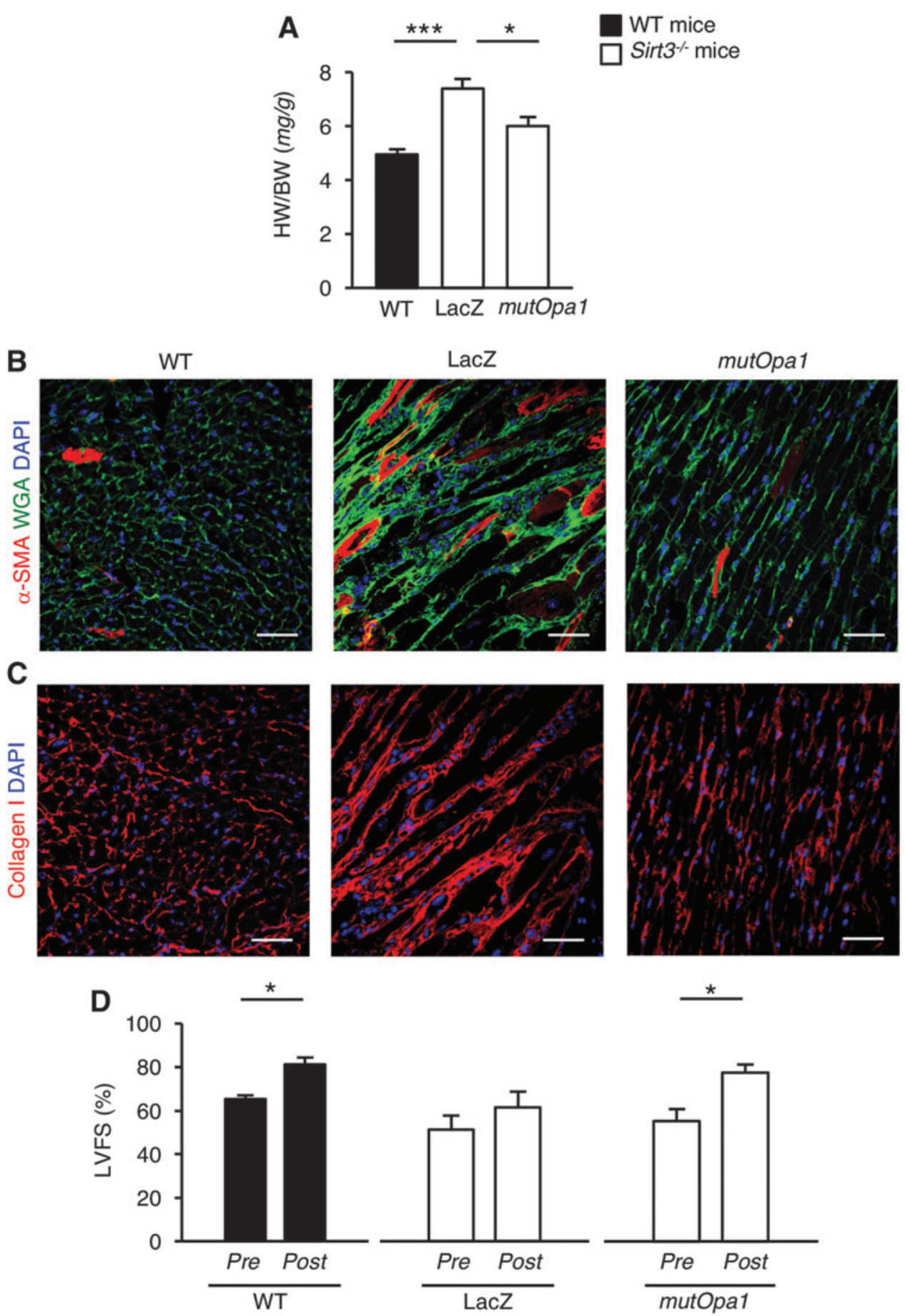

FIG. 6. Long-term effect of mutOpal gene transfer on cardiac structure and function in $\mathrm{Sirt}^{-/-}$mice. (A) HW and BW ratio (HW/BW) in WT mice ( $n=5 /$ group), and Sirt $3^{-1-}$ mice injected with LacZ ( $n=4 /$ group) or mutOpal ( $n=4$ /group). (B) Representative images of $\alpha$-SMA (red) expression in cardiac tissue by immunofluorescence analysis in WT, LacZ, and mutOpal mice. Cardiac structures and nuclei are counterstained with fluorescein isothiocyanate-WGA (green) and DAPI (blue), respectively. Scale bars: $50 \mu \mathrm{m}$. (C) Representative images of collagen I (red) deposition from immunofluorescence analysis in cardiac tissue of WT, LacZ, and mutOpal mice. Cardiac nuclei are counterstained with DAPI (blue). Scale bars: $50 \mu \mathrm{m}$. (D) Assessment of LVFS (LVFS\%) by echocardiography before (Pre) and after (Post) dobutamine stress in WT mice ( $n=5 /$ group), and Sirt ${ }^{-/-}$mice injected with LacZ ( $n=4$ /group) and mutOpal ( $n=4 /$ group). Transfection experiments were performed in Sirt3 $3^{--}$mice at 18-20 months of age, and the analyses were performed 3 months after AAV9 injection. WT age-matched mice were studied as controls. Values are mean \pm SEM. For (A), the $p$-values were derived from ANOVA with Tukey post hoc test. For (D), the $p$-values were derived from paired Student's $t$-test. $* * * p<0.001 ;{ }^{*} p<0.05 . \alpha$-SMA, alphasmooth muscle actin; DAPI, 4',6-diamidino-2-phenylindole; LVFS, left ventricle fractional shortening; WGA, wheat germ agglutinin. 
A

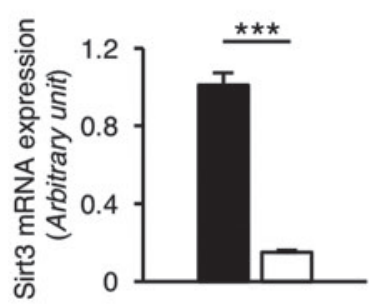

B

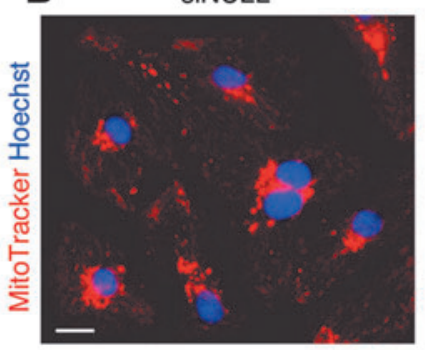



SISIRT3

C
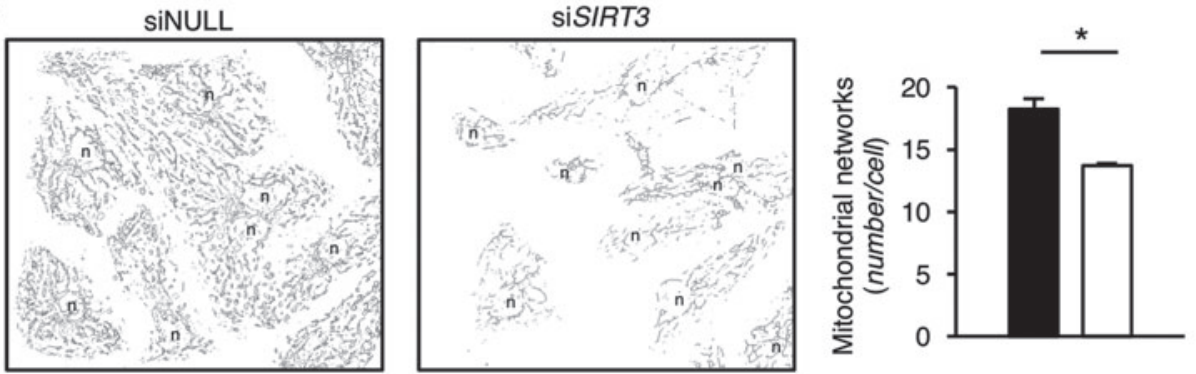
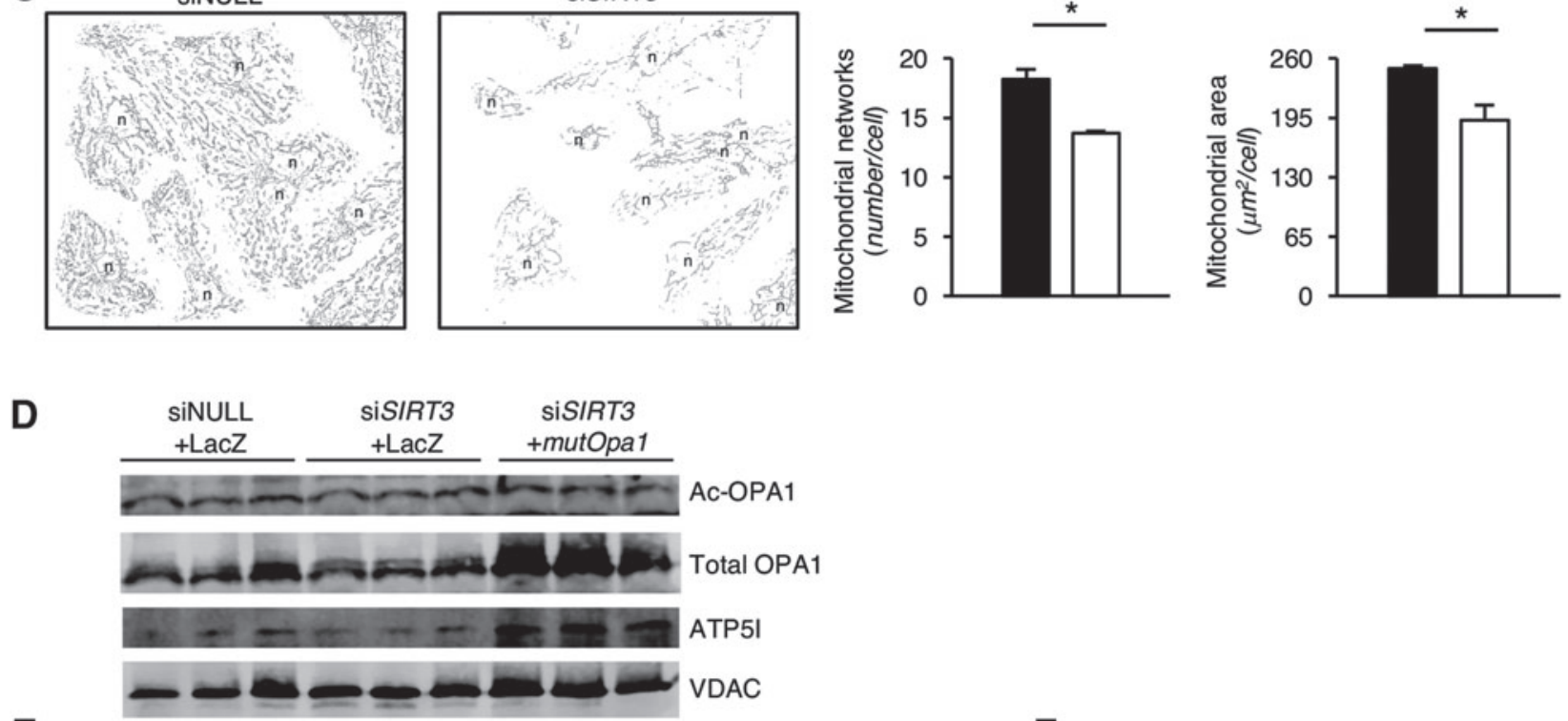

E

$\mathbf{F}$
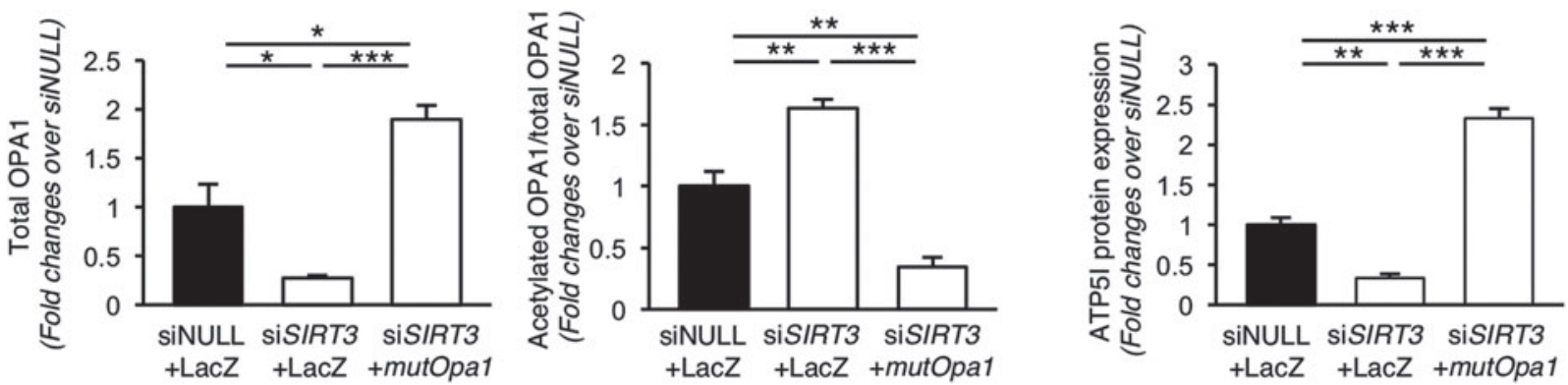

FIG. 7. Effect of mutOpal gene transfer in SIRT3 silenced human pluripotent stem cell-derived cardiomyocytes. (A) Evaluation of SIRT3 mRNA levels in human iPSC-derived cardiomyocytes transfected with a control nontarget small interfering (siNULL) or specific for human SIRT3 (siSIRT3). [(B), left panel] Representative images of mitochondrial distribution visualized by MitoTracker staining (red) in human iPSC-derived cardiomyocytes transfected with siNULL or siSIRT3. Nuclei are counterstained with Hoechst (blue). Scale bar $20 \mu \mathrm{m}$. [(B), right panel] Quantification of mitochondrial perinuclear distribution in iPSC-derived cardiomyocytes transfected with siNULL or siSIRT3 ( $n=3$ independent experiments). (C) Quantification of mitochondrial network and area in iPSC-derived cardiomyocytes transfected with siNULL or siSIRT3 by the Mitochondrial Network Analysis toolset ( $n=3$ independent experiments). Images in (C) are obtained after skeletonization by Fiji software of representative images in (B). $n=$ nuclei. (D-F) Representative gels (D) and densitometric analysis of Western blot of total OPA1 and acetylated (E), and ATP5I (F) in total extracts of siNULL iPSC-derived cardiomyocytes transfected with LacZ and of siSIRT3-silenced iPSC-derived cardiomyocytes transfected with LacZ or mutOpal ( $n=3$ independent experiments). VDAC was used as sample-loading controls. Values are mean \pm SEM. For (A$\mathbf{C})$, the $p$-values were derived from unpaired Student's $t$-test. For $(\mathbf{D}, \mathbf{E})$, the $p$-values were derived from ANOVA with Tukey post hoc test. $* * * p<0.001$; $* p<0.01$; and $* p<0.05$. ATP5I, ATP synthase subunit e; iPSC, induced pluripotent stem cell; siNULL, small interfering RNA null; siSIRT3, small interfering RNA for sirtuin 3. 
has in restoring energy production in $\operatorname{Sirt}^{-/-}$mice could be due to the capability of OPA1 to maintain cristae shape, a prerequisite for the assembly and stability of respiratory chain supercomplexes and hence mitochondrial respiratory efficiency (11). Finding that gene transfer of wtOpal did not affect mitochondrial structure and function, further corroborated the hypothesis that SIRT3 has a critical role in activating OPA1. SIRT3-dependent deacetylation of OPA1 is the only reported posttranslational modification known to promote the activity of OPA1. Our data therefore provide unique proof of principle that modulating the expression of a single gene can have a profound influence on reduced organelle efficiency in Sirt $3^{-/-}$mice. The finding that the correction of OPA1 hyperacetylation via gene transfer sufficed to limit the abnormal myocyte size of Sirt $^{-/-}$mice 2 weeks after mutOpal injection was already an indication that this maneuvre could theoretically preserve cardiac dysfunction.

The next step was to explore the potential of mutOpal gene delivery to mitigate accelerated cardiac hypertrophy, which develops in Sirt $3^{-/-}$mice with age. To actually determine whether this was the case, we performed two sets of experiments based on echocardiography and LDD stress to assess cardiac inotropic reserve (48), given that there is evidence that cardiac inotropic response is a function of mitochondrial integrity and energy production (40). While global systolic function in Sirt $3^{-/-}$and WT mice under resting conditions was the same, the dobutamine challenge unmasked an insufficient contractile response that can reasonably be attributed to the impairment of mitochondrial energetics production in Sirt $3^{-/-}$ mice. Indeed, we found that Sirt $3^{-/-}$mice exhibited reduced Complex I activity in the mitochondrial electron transport chain, with a consequent decrease in $\mathrm{NAD}^{+}$and ATP generation.

These findings may underlie the close link between the SIRT3-dependent OPA1 deacetylation and the preserved spatial distribution of cristae membranes. There is evidence showing that improvement of mitochondrial cristae shape induced by OPA1 ameliorates mitochondrial energetic efficiency (51), as it occurs here with the restoration of Complex I activity after mutOpal gene transfer. This interpretation was confirmed by the finding that these abnormalities were completely overturned by long-term mutOpal gene transfer experiments, which also restored the inotropic response of the heart. It is worth mentioning that deacetylation of OPA1-but not of other mitochondrial proteins including SOD2-maintained in the long term in Sirt $3^{-/-}$animals, corrected cardiac dysfunction and fibrosis, demonstrating this maneuvre's previously unanticipated potential for cardiac protection. Studies have shown that mitochondrial dysfunction in cardiomyocytes critically contributes to the development of age-associated cardiac fibrosis by impairing bioenergetics and reducing cell survival $(1,28,49)$. Furthermore, based on the recent evidence that Complex I inhibition is sufficient to promote the development of fibrosis in the lung, it is tempting to infer that the normalization of Complex I activity through mutOpal gene transfer contributes to the amelioration of cardiac fibrosis (30).

In search of a potential proof-of-concept that SIRT3 deficiency induced cardiomyocyte dysfunction in human specimens as it occurred in mice lacking Sirt3, we sought to study human iPSC-derived cardiomyocytes and used these cells to abrogate SIRT3 expression via specific siRNA. SIRT3 silencing led to altered mitochondrial morphology by reducing the subset of perinuclear mitochondria in iPSC-derived cardiomyocytes. This is significant, given that this mitochondrial clustering is an important factor in ATP generation and the formation of direct energetic channeling between mitochondria and the nucleus $(9,13,22)$, possibly explaining the energetic defect observed in Sirt $3^{-/-}$mice.

On the contrary, SIRT3 also affected the overall cytoplasmic mitochondrial network, which contributes to $\mathrm{Ca}^{2+}$. handling and sustains the proper cardiac contraction (12). In this context, our findings revealed impairment in the iPSCderived cardiomyocyte contractile phenotype associated with altered $\mathrm{Ca}^{2+}$ signaling in the face of reduced SIRT3 expression. We know that human iPSC-derived cardiomyocytes may exhibit several limitations mainly due to their immature phenotype compared with adult cardiomyocytes. However, the novel finding of the present study is that SIRT3 silencing impairs cardiomyocyte contraction and that this phenomenon is fully rescued by restoring OPA1 activity. It is conceivable that our present results explain recent evidence that DNA sequence variants, which actually decrease SIRT3 transcription, play a role in promoting myocardial infarction in humans (54).

Given the theoretical potential for targeting SIRT3 pathways for healthy aging and cardiovascular fitness, this study provides an interesting opportunity to look at gene transfer technology as a therapeutic strategy for the clinical arena in addition to present pharmaceutical treatments. A gene modifying strategy targeting SIRT3 should be especially welcome, in view of the fact that attempts at identifying a specific SIRT3 activator have repeatedly failed.

\section{Materials and Methods}

\section{Animal studies}

Sirt $3^{-/-}$mice generated in a mixed genetic background (provided by Professor Frederick W. Alt, Harvard Medical School, Boston, MA) (25) and their C57BL/6x129 WT littermates were used. Mice were maintained in a specific pathogen-free facility with a 12-h dark/12-h light cycle, in a constant temperature room with free access to standard diet and water. Only male mice were used. Animals were followed up throughout their life span ( $n=25$ for each group). Mice were sacrificed when death appeared imminent, and heart, kidneys, liver, and brain were harvested for tissue analysis. Tumor incidence was determined through histopathological examination of hematoxylin- and eosin-stained slides from the heart, liver, brain and aorta. The oldest WT mice (4/25) were sacrificed at 35.5 months of age for organ collection. Additional WT and Sirt $3^{-/-}$mice were sacrificed at 15 months of age for analysis in cardiac tissues. AAV9LacZ, AAV9-mutOpal, and AAV9-wtOpal gene delivery experiments were performed in Sirt $3^{-/-}$mice at 15 months of age ( $n=3$ /group), and mice sacrificed 2 weeks after AAV9 injection for cardiac analysis. Additional AAV9-LacZ and AAV9-mutOpal gene delivery experiments were performed in Sirt $3^{-1-}$ mice at $18-20$ months $(n=4 /$ group $)$, and mice were sacrificed 3 months after AAV9 injection for cardiac analysis. WT age-matched mice were studied as controls.

All procedures involving animals were performed in accordance with institutional guidelines in compliance with national (D.L.n.26, March 4, 2014) and international laws 
and policies (Directive 2010/63/EU on the protection of animals used for scientific purposes).

\section{Laboratory parameters}

Plasma levels of cholesterol, triglycerides, ALT, and AST were measured by the Reflotron test (Roche Diagnostic Corporation, Indianapolis). Urinary protein excretion was measured through the Comassie method using Cobas Mira autoanalyzer (Roche Diagnostics Systems, Risch, Switzerland).

\section{Cardiac tissue analysis}

Cardiomyocyte cross-sectional areas were evaluated on light microscopy images at $\times 400$ magnification, from at least 50 cells in each group, and measured by manually tracing the cell contour using the Image J analysis program (ImageJ 1.42q) (4). Cardiac tissues were formalin fixed, paraffin embedded, and sectioned at $5 \mu \mathrm{m}$ for hematoxylin/eosin and at $10 \mu \mathrm{m}$ for collagen staining ( $0.1 \%$ Sirius Red in picric acid). Collagen content was quantified in digitally acquired images. The nature of Sirius Red-stained collagen deposits was confirmed by examination under a microscope equipped with polarized light that renders collagen fibers birefringent.

$\alpha$-SMA and collagen I expression was evaluated through immunofluorescence analysis. Frozen heart sections $(3 \mu \mathrm{m})$ were fixed with cold acetone and incubated with $1 \%$ bovine serum albumin (BSA) to block nonspecific sites. Sections were then incubated with cy3-conjugated mouse anti- $\alpha$-SMA (clone 1A4; Sigma-Aldrich, St. Louis, MO) or rabbit anticollagen I (ab34710; Abcam, Cambridge, United Kingdom), the latter followed by Cy3-conjugated goat anti-rabbit immunoglobulin G (IgG, 111-165-144; Jackson Immunoresearch Laboratories, West Grove, PA). Nuclei were stained with 4',6diamidino-2-phenylindole, while fibrotic tissue was labeled with fluorescein isothiocyanate wheat germ agglutinin (Vector Laboratories, Burlingame, CA). The expression of $\alpha$-SMA was quantified as percentage of positive cardiomyocytes, and collagen I signal was graded on a scale of 0-3 (0: no signal, 1: weak, 2: mild, 3 : diffuse), in at least 10-15 randomly selected high-power fields for each animal.

\section{Immunofluorescence analysis of leukocytes in cardiac tissues}

Intragraft leukocytes were analyzed in situ using an indirect immunofluorescence technique on frozen tissue sections $(3 \mu \mathrm{m})$. The sections were incubated with the following antibodies: rat anti-mouse granulocytes (Gr-1, clone RB6-8C5; Biolegend, San Diego, CA) or rat anti-mouse F4/80 (clone BM8; Invitrogen, Carlsbad, CA) for macrophages, both followed by Cy3-conjugated goat anti-rat IgG (112-166-062; Jackson Immunoresearch Laboratories); rat anti-mouse CD4 (clone RM4-5; Biolegend); or rat anti-mouse CD8a (clone 53-6.7; BioLegend) both followed by AF488-conjugated goat anti-rat IgG (A-11006; Invitrogen). For each marker, the number of total positive cells was counted in at least 15-20 randomly selected high-power fields for each animal.

\section{Transmission electron microscopy}

Immediately after excision, cardiac tissue samples were fixed with $2.5 \%$ glutaraldehyde in $0.1 \mathrm{M}$ cacodylate buffer
(pH 7.4) overnight at $4{ }^{\circ} \mathrm{C}$ and postfixed in $1 \%$ osmium tetroxide for $1 \mathrm{~h}$. Then, specimens were dehydrated through ascending grades of alcohol and embedded in Epon resin. Semithin sections $(1 \mu \mathrm{m})$ were stained with toluidine blue in borax and examined by light microscopy. Ultrathin sections $(60-100 \mathrm{~nm})$ were cut on an ultramicrotome (LKB Instruments, Milan, Italy), collected on copper grids, and stained with uranyl acetate and lead citrate. The morphological analysis of ultrastructure was performed using transmission electron microscopy (Morgagni 268D; Philips, Brno, Czech Republic). In total, at least 100 pairs of adjacent mitochondria were analyzed for WT and Sirt $3^{-/}$mice, and mitochondria showing coordination of cristae were counted. In Sirt $3^{-/-}$ mice injected with AAV9-mutOpal, or AAV9-wtOpal, a total of 288 pairs of adjacent mitochondria were studied, for a total of 576 mitochondria.

\section{AAV production and delivery}

A previous study has described that the deacetylation of two Sirt3-targeted lysines (K926, K931) in the C-terminal GTPase effector domain of the human OPA1 sequence (NP_056375.2) results in the activation of OPA1 (41). These two lysines are conserved between human and mouse, and they correspond to the K944 and K949 lysines in the Mus musculus OPA1 dynamin-like $120 \mathrm{kDa}$ protein mitochondrial isoform 1 precursor (NP_001186106.1). The cDNA for the deacetylated form of mouse Opal was synthetized from GenScript. It differs from the wt Opal (GI: 312836757) in two base pair substitutions, c.A2831 > G and c.A2846 > G, and codifies for a protein containing two arginins instead of the conserved lysines K944 and K949 (K to R mutant plasmid). The cDNA was cloned in the AAV vector backbone pZac2.1 (pZac2.1-mutOpal; Gene Therapy Program, Penn Vector core, University of Pennsylvania). To obtain the cDNA for the WT mouse Opal (pZac2.1-wtOpal), site-directed mutagenesis on pZac2.1-mutOpa1 with the QuickChange II XL SiteDirected Mutagenesis Kit (\#200521; Agilent Technologies, Santa Clara, CA) was performed according to the manufacturer's instructions. The following mutagenic primers were used: fwd tgagaagaaggttaaattgctcactggcaaacgagttcagctggc; rev gccagctgaactcgtttgccagtgagcaatttaaccttcttctca. The sequence of the full-length wtOpal was verified by Sanger sequencing on the 3730 DNA Analyzer (Applied Biosystem, Foster City, CA).

pZac2.1-mutOpal and pZac2.1-wtOpal were packaged into AAV capsid serotype 9 (AAV9-mutOpal and AAV9-wtOpal, respectively). Infectious vector stock was prepared by the ICGEB AAV Vector Unit. Briefly, pZac2.1-mutOpal and pZac2.1-wtOpal were cotransfected together with a packaging/helper plasmid into HEK293 cells. Viral stocks were obtained by $\mathrm{CsCl}_{2}$ gradient centrifugation. Titration of AAV viral particles was performed by real-time PCR quantification of the number of viral genomes, measured as cytomegalovirus copy number.

For animal studies, AAV viral vector constructs $\left(1.3 \times 10^{12}\right.$ vector genomes per animal) were diluted in saline to a final volume of $200 \mu \mathrm{L}$ and administered to Sirt $3^{-1-}$ mice at 15 or at 18-20 months of age via a single tail vein injection, and animals were sacrificed 2 weeks or 3 months after AAV9 injection, respectively. 


\section{$X$-Gal staining}

Fresh heart specimens were frozen in optimum cutting temperature compound and stored at $-80^{\circ} \mathrm{C}$. Three-micrometer cryosections were fixed with acetone for $10 \mathrm{~min}$ at $4^{\circ} \mathrm{C}$. X-Gal staining was performed according to the manufacturer's instructions (200384, in situ Beta-Galactosidase Staininig Kit; Stratagene, San Diego, CA). Sections were counterstained with Harris's hematoxylin and mounted in Faramount acqueous mounting medium (S3025; Dako, Santa Clara, CA). Transduction efficiency was expressed as percentage of X-Galpositive cardiomyocytes on the total number of cardiomyocytes, evaluated in 38 random fields from three different areas of the heart.

\section{Citrate synthase activity}

Mitochondrial content in cardiac tissues was evaluated by assessing citrate synthase activity. Citrate synthase activity is an accurate surrogate marker for detecting mitochondrial content in tissues, and compared with other mitochondrial mass indicators, it has the highest positive correlation with morphometric analysis of mitochondria performed with transmission electron microscopy (23). Hearts were washed twice in $0.9 \%(\mathrm{w} / \mathrm{v})$ sodium chloride solution and homogenized in CelLytic MT buffer (C3228; Sigma-Aldrich) supplemented with protease inhibitor cocktail (P8340; SigmaAldrich), and tissue disruption was completed by using a blunt-ended needle and a syringe. As previously described (35), the sample lysates were then centrifuged 16,000 $\mathrm{g}$ for $10 \mathrm{~min}$ at $4^{\circ} \mathrm{C}$ to remove detergent-insoluble material. Supernatants were collected and total protein concentration was determined using DC ${ }^{\mathrm{TM}}$ assay (5000112; Bio-Rad Laboratories, Hercules, CA). Equal amounts of proteins $(10 \mu \mathrm{g})$ were analyzed by citrate synthase activity assay (CS072; Sigma-Aldrich) according to the manufacturer's protocol. The citrate synthase activity was determined by the multimode microplate reader TECAN Infinite M200 ${ }^{\circledR}$ PRO (Tecan Group Ltd., Mannedorf, Switzerland) at $412 \mathrm{~nm}$ under temperature controlled on a kinetic program for $1.5 \mathrm{~min}$ every $10 \mathrm{~s}$.

\section{ATP content assay}

Excised cardiac tissues were washed twice in $0.9 \%(\mathrm{w} / \mathrm{v})$ sodium chloride solution and homogenized in ATP assay buffer (\#K354; BioVision, Milpitas, CA), and tissue disruption was completed by using a blunt-ended needle and a syringe. As previously described (35), the lysates were centrifuged $12,000 \mathrm{~g}$ for $10 \mathrm{~min}$ at $4^{\circ} \mathrm{C}$ to remove detergentinsoluble material. Supernatants were harvested and total protein concentration was determined using the DC assay (5000112; Bio-Rad Laboratories). Then, supernatants were deprived of proteins by using a deproteinizing preparation kit (K808; BioVision) according to the manufacturer's protocol. ATP content assay (K354; BioVision) was then performed on deproteinized samples according to the manufacturer's protocol. The fluorescence intensities were determined by the multimode microplate reader TECAN Infinite M200 PRO (Tecan Group Ltd.) at an excitation wavelength of Ex $535 \mathrm{~nm}$ and emission wavelength of Em $587 \mathrm{~nm}$. Results were normalized for the total protein concentration of each sample.

\section{$N A D^{+}$content assay}

Cardiac tissues were washed twice in $0.9 \%(w / v)$ sodium chloride solution, homogenized in extraction buffer for $\mathrm{NAD}^{+}$ evaluation (KA1657; Abnova, Taipei City, Taiwan), and total protein concentration was determined using DC assay (BioRad Laboratories). As previously described (35), samples were then processed for the determination of the $\mathrm{NAD}^{+}$content by the multimode microplate reader TECAN Infinite M200 PRO (Tecan Group Ltd.) at $565 \mathrm{~nm}$ at a controlled temperature for 15 min according to the manufacturer's protocol. Results were normalized for total protein concentration of each sample.

\section{Complex I activity assay}

Cardiac tissues were washed twice in $0.9 \%(w / v)$ sodium chloride solution, homogenized in detergent solution according to the manufacturer's instructions (ab109721 Assay Kit; Abcam), and the total protein concentration was determined using DC assay (Bio-Rad Laboratories). A total of $20 \mu \mathrm{g}$ of heart extracts was then processed for the determination of Complex I activity according to the manufacturer's instructions (ab109721 Assay Kit; Abcam). Complex I activity in each sample was determined by the multimode microplate reader TECAN Infinite M200 PRO (Tecan Group Ltd.) at $450 \mathrm{~nm}$ under a kinetic program at a controlled temperature for 30 min according to the manufacturer's instructions.

\section{Cardiac function and dobutamine stress test}

Cardiac function in mice anesthetized with 2 vol\% isoflurane in $0.5 \mathrm{~L} / \mathrm{min} \mathrm{O}_{2}$ was assessed by transthoracic echocardiography using a commercially available echocardiographer equipped with a 12-MHz probe (MyLab30; Esaote, Geneva, Italy), as previously described (44). Warmed echo gel was placed on the shaved chest and each parameter was measured using B-mode-guided M-mode imaging in the parasternal short-axis view at the level of the papillary muscles. Images were analyzed off-line in a blinded manner. The global cardiac function was calculated as the percentage of fractional shortening (LVFS \%) of the left ventricle. Heart rate and rhythm were simultaneously monitored by three-lead electrocardiogram (ECG). The cardiac function was evaluated at rest and at peak after intraperitoneal injection of a single bolus of dobutamine $(1.5 \mu \mathrm{g} / \mathrm{g}$ of body weight), an established method for assessing the myocardial contractile reserve $(10,48)$. Echocardiographic scans and ECG recordings were repeated immediately after a single bolus injection and then periodically over $10 \mathrm{~min}$, until the peak heart rate response was reached and heart rate began to decline again. ECG was recorded for $3 \mathrm{~min}$ at rest and at the peak of dobutamine stress.

\section{Human iPSC-derived cardiomyocyte culture}

Human iPSC-derived cardiomyocytes (C1006; Cellular Dynamics International, Madison, WI) were seeded on $0.1 \%$ gelatin-coated wells and cultured in a plating medium (M1001; Cellular Dynamics International) at a density of 45,000 viable cells $/ \mathrm{cm}^{2}$. At day 2, the medium was replaced with maintenance medium (M1003; Cellular Dynamics International) and changed at day 4 and 6 . 


\section{SIRT3 silencing and deacetylated Opa1 transfer}

On day 7 postplating, iPSC-derived cardiomyocytes were transfected with $50 \mathrm{nM}$ Silencer Select predesigned siRNA human SIRT3 (siSIRT3, s23768; Life Technologies, Carlsbad, CA) or control nontarget siRNA (siNULL, Silencer Select Negative Control \#2 siRNA; Ambion, Carlsbad, CA) using the TransIT-KO Transfection Reagent (MIR2150; Mirus Bio, Madison, WI) according to the Cellular Dynamics International instructions. After $48 \mathrm{~h}$ of transfection, cells were used for subsequent experiments.

In selected experiments, iPSC-derived cardiomyocytes were transduced with $2 \times 10^{6}$ multiplicity of infection/cells of AAV9-mutOpal and 2 days later, SIRT3 silencing was performed as above. AAV9-LacZ- transduced iPSC-derived cardiomyocytes transfected with siNULL were used as control. On day 5 posttransduction, iPSC-derived cardiomyocytes were used for the analysis of cell contractility and total protein extraction.

\section{Real-time PCR}

Human iPSC-derived cardiomyocytes were harvested in TRIzol reagent (15596026; Invitrogen, Life Technologies), and total RNA was extracted according to the manufacturer's instructions. Contaminating genomic DNA was removed by RNase-free DNase (M6101; Promega, Madison, WI) for $1 \mathrm{~h}$ at $37^{\circ} \mathrm{C}$. The first-strand cDNA $(2.5 \mu \mathrm{g})$ was produced using a SuperScript VILO cDNA Synthesis Kit (11754050; Life Technologies) according to the manufacturer's instruction. To amplify cDNA of human SIRT3, we used the TaqMan Universal PCR Master Mix (4304437; Applied Biosystems) and inventoried TaqMan assays of the SIRT3 gene (FAMMBG probe Hs00202030_m1), and of the human HPRT endogenous control (FAM-MGB probe, Hs99999909_m1) according to the manufacturer's instructions. PCR was performed on the Viia7 Real-Time PCR System (Applied Biosystems). Data were analyzed using the $2^{-\Delta \Delta C T}$ method and presented as fold changes relative to siNULL cells.

\section{MitoTracker analysis}

To evaluate mitochondrial morphology, living iPSCderived cardiomyocytes were incubated with a $250 \mathrm{nM}$ fluorescent probe MitoTracker ${ }^{\circledR}$ Red (M7512; Thermo Fisher, Invitrogen) for $30 \mathrm{~min}$, at $37^{\circ} \mathrm{C}, 5 \% \mathrm{CO}_{2}$. Nuclei were counterstained with NucBlue ${ }^{\circledR}$ Live ReadyProbes ${ }^{\circledR}$ (Hoechst; R37605; Thermo Fisher, Invitrogen) according to the manufacturer's protocol. At the end of the probe incubations, living cells were examined using the Cell Observer system (Axio Observer.Z1; Zeiss Oberkochen, Germania) and mitochondrial morphology was evaluated using the Mitochondrial Network Analysis toolset on Fiji software (50). Data were normalized for the number of Hoechst-positive cells in 10 random fields per sample.

\section{Cell contractility and FLUO-4 analysis}

To detect cell contractility, living iPSC-derived cardiomyocytes were seeded on 35-mm glass-bottomed dishes, monitored under phase contrast using the Cell Observer system (Axio Observer.Z1; Zeiss), and stably recorded for at least $2 \mathrm{~min}$ in different fields of the dish. For calcium analysis, the nonratiometric Fluo-4 dye Calcium Imaging Kit was used (F10489; Thermo Fisher, Invitrogen), according to the manufacturer's protocol. Briefly, living iPSC-derived cardiomyocytes were incubated with Fluo-4 AM in Live Cell Imaging Solution (Thermo Fisher, Invitrogen) supplemented with $20 \mathrm{mM}$ glucose (Sigma-Aldrich) for $30 \mathrm{~min}$ at $37^{\circ} \mathrm{C}, 5 \%$ $\mathrm{CO}_{2}$. At the end of probe incubations, living cells were rinsed with the iCell Cardiomyocytes Maintenance Medium (M1003; Cellular Dynamics International) and recorded for at least $2 \mathrm{~min}$ in different fields of the dish by the Cell Observer system (Axio Observer.Z1; Zeiss).

\section{Mitochondrial isolation from cardiac tissue}

Mitochondria were isolated from mouse cardiac tissue using the Qproteome Mitochondria Isolation Kit (37612; Qiagen S.r.l., Milan, Italy) according to the manufacturer's protocol. Isolated mitochondria were solubilized in mitochondrial storage buffer (37612; Qiagen S.r.1.) and total protein concentration was determined using the DC assay (5000112; Bio-Rad Laboratories).

\section{Protein extraction from human iPSC-derived cardiomyocytes}

For total protein extraction, human iPSC-derived cardiomyocytes were washed twice in $0.9 \%(\mathrm{w} / \mathrm{v})$ sodium chloride solution and lysed by sonication in mammalian CelLytic $\mathrm{M}$ lysis buffer (C2978; Sigma-Aldrich) supplemented with protease inhibitor cocktail (P8340; Sigma-Aldrich). After sonication, the sample lysates were centrifuged $16,000 \mathrm{~g}$ for $10 \mathrm{~min}$ at $4^{\circ} \mathrm{C}$, and protein concentration was determined using DC assay (Bio-Rad Laboratories).

\section{Evaluation of OPA1 and SOD2 acetylation}

OPA1 and SOD2 acetylation was determined by standard Western blot analysis. Briefly, equal amounts of mitochondrial proteins from cardiac tissue $(30 \mu \mathrm{g})$ or total extracts of iPSC-derived cardiomyocytes $(20 \mu \mathrm{g})$ were separated on $12 \%$ sodium dodecyl sulfate (SDS)/polyacrylamide gel electrophoresis (PAGE) under reducing conditions and transferred to nitrocellulose membranes (Bio-Rad Laboratories). After blocking with 5\% BSA in Tris-buffered saline (TBS) supplemented with $0.1 \%$ Tween-20, membranes were incubated overnight at $4^{\circ} \mathrm{C}$ with the following antibodies: rabbit anti-acetylated-lysine (1:1000, BK9441; Cell Signaling) and mouse anti-OPA1 antibody (1:1000, 612606; BD Bioscience) or sheep anti-SOD2 (1:1000, 574596; Merck Millipore). The signals were visualized on an Odyssey ${ }^{\circledR}$ FC Imaging System (LiCor, Lincoln, NE) by infrared (IR) fluorescence using a secondary goat anti-rabbit IRDye 680LT antibody $(1: 1000$ FE3680210; LiCor) and a goat anti-mouse IRDye $800 \mathrm{CW}$ (1:1000FE30926210; LiCor) or by enhanced chemiluminescenceWestern blotting detection reagent (Pierce, Thermo Fisher Scientific, Inc.) using donkey anti-sheep (1:10,000 A3415; Sigma-Aldrich) horseradish peroxidase secondary antibodies. Mouse anti-VDAC (voltage-dependent anion channel [VDAC], 1:2000ab186321; Abcam) was used as sample-loading controls in isolated mitochondria of the same membranes. Bands were quantified by densitometry using the Image Studio Lite 5.0 (LiCor) software. OPA1 and SOD2 acetylation was expressed as the ratio between the band of acetylated lysine colocalizing with the band corresponding to total OPA1 or SOD2. Total OPA1 protein level was expressed as the ratio 
between the bands of OPA1 and VDAC. For any cropped Western blot shown in the main body of the article, the full uncut gels are found in Supplementary Figure S5.

\section{Western blot analysis of ATP5I}

Equal amounts of total extracts of iPSC-derived cardiomyocytes $(20 \mu \mathrm{g})$ were separated on $12 \%$ SDS-PAGE under reducing conditions and transferred to nitrocellulose membranes (Bio-Rad Laboratories). After blocking with 5\% BSA in TBS supplemented with $0.1 \%$ Tween-20, membranes were incubated with a rabbit anti-ATP5I antibody (1:1000 HPA035010; Sigma-Aldrich). Mouse anti-VDAC (1:2000ab186321; Abcam) was used as sample-loading controls in isolated mitochondria of the same membranes. The signals were visualized on an Odyssey ${ }^{\circledR} \mathrm{FC}$ Imaging System (LiCor) by IR fluorescence using a secondary goat anti-rabbit IRDye 680LT antibody (FE3680210; LiCor) and a goat anti-mouse IRDye $800 \mathrm{CW}$ (FE30926210; LiCor), as appropriate. Bands were quantified by densitometry using the Image Studio Lite (LiCor) 5.0 software. Total ATP5I protein level was expressed as the ratio between the bands of ATP5I and VDAC. For any cropped Western blot shown in the main body of the article, the full uncut gels are found in Supplementary Figure S5.

\section{Statistics}

The significance level of difference between individual groups was analyzed by the unpaired or paired Student's $t$-test, or analysis of variance with Tukey post hoc test comparisons, and the statistical significance was defined as $p$-value of $<0.05$. Results are given as mean \pm standard error of the mean (SEM).

Statistics for life span comparison between Sirt $3^{-/-}$and WT mice was evaluated through log-rank calculations. Maximum life span of Sirt $3^{-1-}$ and WT mice was compared by means of a Laplace regression, considering the time by which $90 \%$ of the mice had died (90th percentile of survival). The 'Laplace' package for STATA version 11 was retrieved for this purpose.

For the analysis of risk of cristae alignment alterations, odds ratios with their $95 \%$ confidence intervals were estimated taking into account familial dependence between observations with generalized estimated equations using the GENMOD procedure in $\mathrm{SAS}^{\circledR}$, allowing the extension of traditional linear model theory to generalized linear models by allowing the mean of a population to depend on a linear predictor through a nonlinear link function. In this article, we investigated a binary outcome modeling approach using PROC GENMOD with the link functions and aggregate, with post hoc Bonferroni correction. Results are given as mean \pm SEM, and the statistical significance was adjusted for multiple comparisons and was defined as $p$-value of $<0.005$.

\section{Acknowledgments}

The authors thank Sebastian Villa and Fabrizio Giordano for the animal study, Paola Rizzo for technical assistance with immunostaining, and Prof. Gino Villetti for histologic analysis. They thank Manuela Passera for helping to prepare the article and Kerstin Mierke for English language editing. L.P. is the recipient of a fellowship from Fondazione Aiuti per la Ricerca sulle Malattie Rare (ARMR), Bergamo, Italy. Do.C. is the recipient of a fellowship from Fondazione Istituti Educativi di Bergamo, Bergamo, Italy.

\section{Authors' Contributions}

A.B. and G.R. designed the study, discussed the data, and wrote the article; P.C. and S.C. designed the study, performed the experiments, discussed the data, and contributed to writing the article; L.P. performed the experiments, discussed the data, and contributed to writing the article; Do.C. and Da.C. performed the in vivo experiments; L.Z. generated the AAV9 vectors; P.T. performed the experiments; C.Z. discussed the data and contributed to writing the article; A.P. performed statistical analysis; V.L. discussed the data and performed measurements of cardiac function; M.G. discussed the data and contributed to writing the article; S.T. contributed to the research plan, discussed the data, and contributed to writing the article. All authors commented on the article.

\section{Author Disclosure Statement}

No competing financial interests exist.

\section{Supplementary Material}

Supplementary Figure S1

Supplementary Figure S2

Supplementary Figure S3

Supplementary Figure S4

Supplementary Figure S5

Supplementary Table S1

Supplementary Movie S1

Supplementary Movie S2

Supplementary Movie S3

Supplementary Movie S4

Supplementary Movie S5

Supplementary Movie S6

Supplementary Movie S7

\section{References}

1. Abdullah CS, Alam S, Aishwarya R, Miriyala S, Panchatcharam M, Bhuiyan MAN, Peretik JM, Orr AW, James J, Osinska H, Robbins J, Lorenz JN, and Bhuiyan MS. Cardiac dysfunction in the sigma 1 receptor knockout mouse associated with impaired mitochondrial dynamics and bioenergetics. J Am Heart Assoc 7: e009775, 2018.

2. Ahn BH, Kim HS, Song S, Lee IH, Liu J, Vassilopoulos A, Deng CX, and Finkel T. A role for the mitochondrial deacetylase Sirt3 in regulating energy homeostasis. Proc Natl Acad Sci U S A 105: 14447-14452, 2008.

3. Amchenkova AA, Bakeeva LE, Chentsov YS, Skulachev VP, and Zorov DB. Coupling membranes as energytransmitting cables. I. Filamentous mitochondria in fibroblasts and mitochondrial clusters in cardiomyocytes. $J$ Cell Biol 107: 481-495, 1988.

4. Anversa P, Beghi C, Kikkawa Y, and Olivetti G. Myocardial response to infarction in the rat. Morphometric measurement of infarct size and myocyte cellular hypertrophy. Am J Pathol 118: 484-492, 1985.

5. Benigni A, Corna D, Zoja C, Sonzogni A, Latini R, Salio M, Conti S, Rottoli D, Longaretti L, Cassis P, Morigi M, Coffman TM, and Remuzzi G. Disruption of the Ang II type 1 receptor promotes longevity in mice. J Clin Invest 119: 524-530, 2009.

6. Benigni A, Perico L, and Macconi D. Mitochondrial dynamics is linked to longevity and protects from end-organ 
injury: the emerging role of sirtuin 3. Antioxid Redox Signal 25: 185-199, 2016.

7. Bleck CKE, Kim Y, Willingham TB, and Glancy B. Subcellular connectomic analyses of energy networks in striated muscle. Nat Commun 9: 5111, 2018.

8. Bokov AF, Lindsey ML, Khodr C, Sabia MR, and Richardson A. Long-lived ames dwarf mice are resistant to chemical stressors. J Gerontol A Biol Sci Med Sci 64: 819-827, 2009.

9. Bruce JI, Giovannucci DR, Blinder G, Shuttleworth TJ, and Yule DI. Modulation of [Ca2+]i signaling dynamics and metabolism by perinuclear mitochondria in mouse parotid acinar cells. J Biol Chem 279: 12909-12917, 2004.

10. Cassis P, Cerullo D, Zanchi C, Corna D, Lionetti V, Giordano F, Novelli R, Conti S, Casieri V, Matteucci M, Locatelli M, Taraboletti G, Villa S, Gastoldi S, Remuzzi G, Benigni A, and Zoja C. ADAMTS13 deficiency shortens the life span of mice with experimental diabetes. Diabetes 67: 2069-2083, 2018.

11. Civiletto G, Varanita T, Cerutti R, Gorletta T, Barbaro S, Marchet S, Lamperti C, Viscomi C, Scorrano L, and Zeviani M. Opa1 overexpression ameliorates the phenotype of two mitochondrial disease mouse models. Cell Metab 21: 845854, 2015.

12. Contreras L, Drago I, Zampese E, and Pozzan T. Mitochondria: the calcium connection. Biochim Biophys Acta 1797: 607-618, 2010.

13. Dzeja PP, Bortolon R, Perez-Terzic C, Holmuhamedov EL, and Terzic A. Energetic communication between mitochondria and nucleus directed by catalyzed phosphotransfer. Proc Natl Acad Sci U S A 99: 10156-10161, 2002.

14. Finley LW, Haas W, Desquiret-Dumas V, Wallace DC, Procaccio V, Gygi SP, and Haigis MC. Succinate dehydrogenase is a direct target of sirtuin 3 deacetylase activity. PLoS One 6: e23295, 2011.

15. Hafner AV, Dai J, Gomes AP, Xiao CY, Palmeira CM, Rosenzweig A, and Sinclair DA. Regulation of the mPTP by SIRT3-mediated deacetylation of CypD at lysine 166 suppresses age-related cardiac hypertrophy. Aging (Albany NY) 2: 914-923, 2010.

16. Hebert AS, Dittenhafer-Reed KE, Yu W, Bailey DJ, Selen ES, Boersma MD, Carson JJ, Tonelli M, Balloon AJ, Higbee AJ, Westphall MS, Pagliarini DJ, Prolla TA, Assadi-Porter F, Roy S, Denu JM, and Coon JJ. Calorie restriction and SIRT3 trigger global reprogramming of the mitochondrial protein acetylome. Mol Cell 49: 186-199, 2013.

17. Hirschey MD, Shimazu T, Goetzman E, Jing E, Schwer B, Lombard DB, Grueter CA, Harris C, Biddinger S, Ilkayeva OR, Stevens RD, Li Y, Saha AK, Ruderman NB, Bain JR, Newgard CB, Farese RV, Jr., Alt FW, Kahn CR, and Verdin E. SIRT3 regulates mitochondrial fatty-acid oxidation by reversible enzyme deacetylation. Nature 464: 121-125, 2010.

18. Hirschey MD, Shimazu T, Jing E, Grueter CA, Collins AM, Aouizerat B, Stancakova A, Goetzman E, Lam MM, Schwer B, Stevens RD, Muehlbauer MJ, Kakar S, Bass NM, Kuusisto J, Laakso M, Alt FW, Newgard CB, Farese RV, Jr., Kahn CR, and Verdin E. SIRT3 deficiency and mitochondrial protein hyperacetylation accelerate the development of the metabolic syndrome. Mol Cell 44: 177190, 2011

19. Houtkooper RH, Pirinen E, and Auwerx J. Sirtuins as regulators of metabolism and healthspan. Nat Rev Mol Cell Biol 13: 225-238, 2012.

20. Kim HS, Patel K, Muldoon-Jacobs K, Bisht KS, AykinBurns N, Pennington JD, van der Meer R, Nguyen P, Sa- vage J, Owens KM, Vassilopoulos A, Ozden O, Park SH, Singh KK, Abdulkadir SA, Spitz DR, Deng CX, and Gius D. SIRT3 is a mitochondria-localized tumor suppressor required for maintenance of mitochondrial integrity and metabolism during stress. Cancer Cell 17: 41-52, 2010.

21. Klecker T, Bockler S, and Westermann B. Making connections: interorganelle contacts orchestrate mitochondrial behavior. Trends Cell Biol 24: 537-545, 2014.

22. Kuznetsov AV, Troppmair J, Sucher R, Hermann M, Saks V, and Margreiter R. Mitochondrial subpopulations and heterogeneity revealed by confocal imaging: possible physiological role? Biochim Biophys Acta 1757: 686-691, 2006.

23. Larsen S, Nielsen J, Hansen CN, Nielsen LB, Wibrand F, Stride N, Schroder HD, Boushel R, Helge JW, Dela F, and Hey-Mogensen M. Biomarkers of mitochondrial content in skeletal muscle of healthy young human subjects. $J$ Physiol 590: 3349-3360, 2012.

24. Li Y, Ma Y, Song L, Yu L, Zhang L, Zhang Y, Xing Y, Yin $\mathrm{Y}$, and Ma H. SIRT3 deficiency exacerbates p53/Parkin mediated mitophagy inhibition and promotes mitochondrial dysfunction: implication for aged hearts. Int J Mol Med 41: 3517-3526, 2018.

25. Lombard DB, Alt FW, Cheng HL, Bunkenborg J, Streeper RS, Mostoslavsky R, Kim J, Yancopoulos G, Valenzuela D, Murphy A, Yang Y, Chen Y, Hirschey MD, Bronson RT, Haigis M, Guarente LP, Farese RV, Jr., Weissman S, Verdin E, and Schwer B. Mammalian Sir2 homolog SIRT3 regulates global mitochondrial lysine acetylation. Mol Cell Biol 27: 8807-8814, 2007.

26. Lombard DB and Zwaans BM. SIRT3: as simple as it seems? Gerontology 60: 56-64, 2014.

27. Lu JY, Lin YY, Zhu H, Chuang LM, and Boeke JD. Protein acetylation and aging. Aging (Albany NY) 3: 911-912, 2011.

28. Martin-Fernandez B and Gredilla R. Mitochondria and oxidative stress in heart aging. Age (Dordr) 38: 225-238, 2016.

29. McDonnell E, Peterson BS, Bomze HM, and Hirschey MD. SIRT3 regulates progression and development of diseases of aging. Trends Endocrinol Metab 26: 486-492, 2015.

30. Mora AL, Bueno M, and Rojas M. Mitochondria in the spotlight of aging and idiopathic pulmonary fibrosis. J Clin Invest 127: 405-414, 2017.

31. Morigi M, Perico L, Rota C, Longaretti L, Conti S, Rottoli D, Novelli R, Remuzzi G, and Benigni A. Sirtuin 3-dependent mitochondrial dynamic improvements protect against acute kidney injury. J Clin Invest 125: 715-726, 2015.

32. Ogura Y, Kitada M, Monno I, Kanasaki K, Watanabe A, and Koya D. Renal mitochondrial oxidative stress is enhanced by the reduction of Sirt3 activity, in Zucker diabetic fatty rats. Redox Rep 23: 153-159, 2018.

33. Onyango P, Celic I, McCaffery JM, Boeke JD, and Feinberg AP. SIRT3, a human SIR2 homologue, is an NADdependent deacetylase localized to mitochondria. Proc Natl Acad Sci U S A 99: 13653-13658, 2002.

34. Perico L, Morigi M, and Benigni A. Mitochondrial sirtuin 3 and renal diseases. Nephron 134: 14-19, 2016.

35. Perico L, Morigi M, Rota C, Breno M, Mele C, Noris M, Introna M, Capelli C, Longaretti L, Rottoli D, Conti S, Corna D, Remuzzi G, and Benigni A. Human mesenchymal stromal cells transplanted into mice stimulate renal tubular cells and enhance mitochondrial function. Nat Commun 8: 983, 2017.

36. Picard M. Mitochondrial synapses: intracellular communication and signal integration. Trends Neurosci 38: 468-474, 2015. 
37. Picard M, McManus MJ, Csordas G, Varnai P, Dorn GW, 2nd, Williams D, Hajnoczky G, and Wallace DC. Transmitochondrial coordination of cristae at regulated membrane junctions. Nat Commun 6: 6259, 2015.

38. Quintana-Cabrera R, Quirin C, Glytsou C, Corrado M, Urbani A, Pellattiero A, Calvo E, Vazquez J, Enriquez JA, Gerle C, Soriano ME, Bernardi P, and Scorrano L. The cristae modulator Optic atrophy 1 requires mitochondrial ATP synthase oligomers to safeguard mitochondrial function. Nat Commun 9: 3399, 2018.

39. Rahman M, Nirala NK, Singh A, Zhu LJ, Taguchi K, Bamba T, Fukusaki E, Shaw LM, Lambright DG, Acharya JK, and Acharya UR. Drosophila Sirt2/mammalian SIRT3 deacetylates ATP synthase beta and regulates complex V activity. J Cell Biol 206: 289-305, 2014.

40. Rosca MG, Tandler B, and Hoppel CL. Mitochondria in cardiac hypertrophy and heart failure. J Mol Cell Cardiol 55: 31-41, 2013.

41. Samant SA, Zhang HJ, Hong Z, Pillai VB, Sundaresan NR, Wolfgeher D, Archer SL, Chan DC, and Gupta MP. SIRT3 deacetylates and activates OPA1 to regulate mitochondrial dynamics during stress. Mol Cell Biol 34: 807-819, 2014.

42. Schwer B, North BJ, Frye RA, Ott M, and Verdin E. The human silent information regulator (Sir)2 homologue hSIRT3 is a mitochondrial nicotinamide adenine dinucleotidedependent deacetylase. J Cell Biol 158: 647-657, 2002.

43. Someya S, Yu W, Hallows WC, Xu J, Vann JM, Leeuwenburgh C, Tanokura M, Denu JM, and Prolla TA. Sirt3 mediates reduction of oxidative damage and prevention of age-related hearing loss under caloric restriction. Cell 143: 802-812, 2010.

44. Stypmann J, Engelen MA, Epping C, van Rijen HV, Milberg P, Bruch C, Breithardt G, Tiemann K, and Eckardt L. Age and gender related reference values for transthoracic Doppler-echocardiography in the anesthetized CD1 mouse. Int J Cardiovasc Imaging 22: 353-362, 2006.

45. Sundaresan NR, Bindu S, Pillai VB, Samant S, Pan Y, Huang JY, Gupta M, Nagalingam RS, Wolfgeher D, Verdin E, and Gupta MP. SIRT3 blocks aging-associated tissue fibrosis in mice by deacetylating and activating glycogen synthase kinase 3beta. Mol Cell Biol 36: 678-692, 2016.

46. Sundaresan NR, Gupta M, Kim G, Rajamohan SB, Isbatan A, and Gupta MP. Sirt3 blocks the cardiac hypertrophic response by augmenting Foxo3a-dependent antioxidant defense mechanisms in mice. J Clin Invest 119: 2758-2771, 2009.

47. Szabadkai G, Simoni AM, Bianchi K, De Stefani D, Leo S, Wieckowski MR, and Rizzuto R. Mitochondrial dynamics and Ca2+ signaling. Biochim Biophys Acta 1763: 442-449, 2006.

48. Tanaka N, Dalton N, Mao L, Rockman HA, Peterson KL, Gottshall KR, Hunter JJ, Chien KR, and Ross J, Jr. Transthoracic echocardiography in models of cardiac disease in the mouse. Circulation 94: 1109-1117, 1996.

49. Torrealba N, Aranguiz P, Alonso C, Rothermel BA, and Lavandero S. Mitochondria in structural and functional cardiac remodeling. Adv Exp Med Biol 982: 277-306, 2017.

50. Valente AJ, Maddalena LA, Robb EL, Moradi F, and Stuart JA. A simple ImageJ macro tool for analyzing mitochondrial network morphology in mammalian cell culture. Acta Histochem 119: 315-326, 2017.

51. Varanita T, Soriano ME, Romanello V, Zaglia T, QuintanaCabrera R, Semenzato M, Menabo R, Costa V, Civiletto G, Pesce P, Viscomi C, Zeviani M, Di Lisa F, Mongillo M, Sandri M, and Scorrano L. The OPA1-dependent mito- chondrial cristae remodeling pathway controls atrophic, apoptotic, and ischemic tissue damage. Cell Metab 21: 834-844, 2015.

52. Wagner GR and Payne RM. Mitochondrial acetylation and diseases of aging. J Aging Res 2011: 234875, 2011.

53. Wood JG, Schwer B, Wickremesinghe PC, Hartnett DA, Burhenn L, Garcia M, Li M, Verdin E, and Helfand SL. Sirt4 is a mitochondrial regulator of metabolism and lifespan in Drosophila melanogaster. Proc Natl Acad Sci U S A 115: 1564-1569, 2018.

54. Yin X, Pang S, Huang J, Cui Y, and Yan B. Genetic and functional sequence variants of the SIRT3 gene promoter in myocardial infarction. PLoS One 11: e0153815, 2016.

55. Zacchigna S, Zentilin L, and Giacca M. Adeno-associated virus vectors as therapeutic and investigational tools in the cardiovascular system. Circ Res 114: 1827-1846, 2014.

Address correspondence to: Dr. Ariela Benigni

Istituto di Ricerche Farmacologiche Mario Negri IRCCS Centro Anna Maria Astori Science and Technology Park Kilometro Rosso Via Stezzano, 87 Bergamo 24126 Italy

E-mail: ariela.benigni@marionegri.it

Date of first submission to ARS Central, November 30, 2018; date of final revised submission, July 2, 2019; date of acceptance, July 3, 2019.

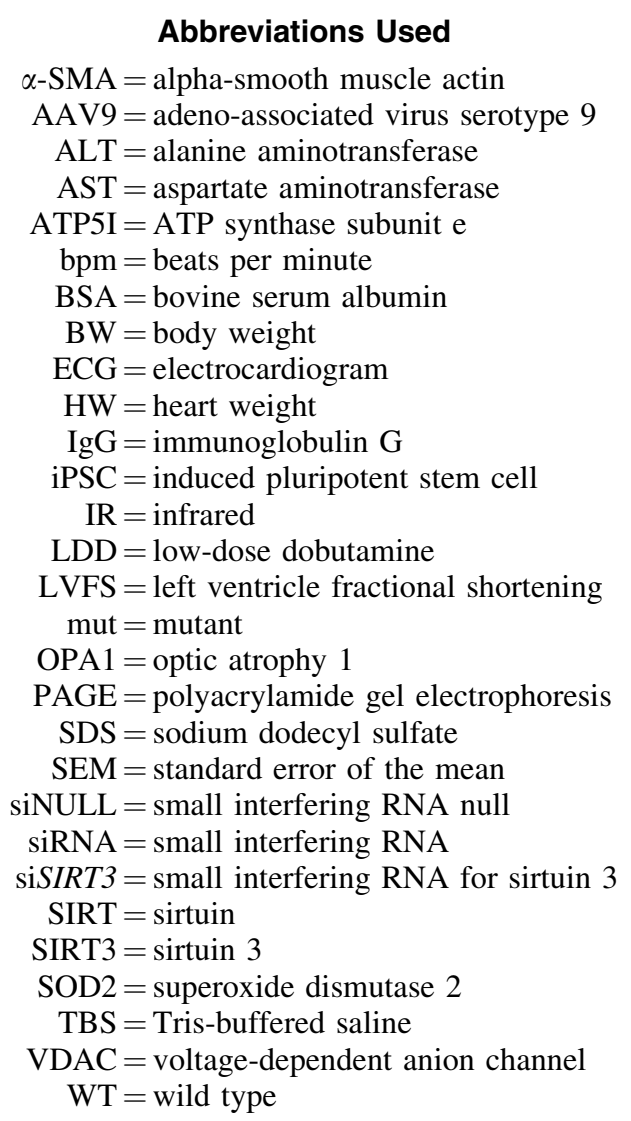

